Optinalysis

\title{
Optinalysis: A New Method of Data Analysis and Comparison
}

\author{
*Kabir Bindawa Abdullahi \\ Department of Biology, Faculty of Natural and Applied Sciences, Umaru Musa Yar'adua University, P.M.B., \\ 2218 Katsina, Katsina State, Nigeria. \\ *Correspondence: kabir.abdullahi@umyu.edu.ng ; kabirnamallam@gmail.com \\ ORCID: 0000-0002-3810-9592
}

\begin{abstract}
The key concepts in symmetry detection and similarity, identity measures are automorphism and isomorphism respectively. Therefore, methods for symmetry detection and similarity, identity measures should be functionally bijective, inverse, and invariance under a set of mathematical operations. Nevertheless, few or no existing method is functional for these properties. In this paper, a new methodological paradigm, called optinalysis, is presented for symmetry detections, similarity, and identity measures between isoreflective or autoreflective pair of mathematical structures. The paradigm of optinalysis is the re-mapping of isoreflective or autoreflective pairs with an optical scale. Optinalysis is characterized as invariant under a set of transformations and its isoreflective polymorphism behaves on polynomial and non-polynomial models.
\end{abstract}

Keywords: Autoreflectivity; Identity; Isoreflectivity; Kabirian coefficient; Similarity; Symmetry.

\section{Introduction}

The notion of isometry (as a congruence mapping) is a general phenomenon commonly accepted in Mathematics. It means a mapping that preserves distances. It is a bijective mapping, characterized as one-toone mapping of a group onto itself or onto another in various transformational ways such as reflections, translation, or rotations [1].

Two graphs are isomorphic if there is a bijection between the mathematical structures that preserves adjacency; such a bijection is called an isomorphism. In other terms, two graphs $A$ and $B$ as isomorphic if they have the same structure, but their elements or vertices may be different [2]. An isomorphism from a graph onto itself is called an automorphism, and the set of all automorphisms of a given graph $G$ denoted $A u t(G)$, forms a group under composition [2].

Methods of symmetry/asymmetry detection of shapes and distributions (e.g: Root mean squared error (RMSE), and Areal ratio (AR), Pearson's first and second coefficients of skewness, Yule's coefficient of skewness, the standardized third central moment, Bowley's coefficient of skewness, and three Gallip's coefficients of skewness); and methods of similarity/dissimilarity/distances measures of shapes and distributions (eg: Cosine, Morisita, Horn, Correlation, Rho, Dice, Jaccard, Ochiai, Kulczynski, Simpson, BrayCurtis methods, Raup-Crick, and Riemannian distance, Euclidean distance, Manhattan distance, Kimura, Chord, Gower and Hamming/P-distances) have been developed since earlier time. But most of the proposals are ad-hoc and only a few, if any, can be theoretically proven in line with the theorems of automorphism and isomorphism.

In this paper, optinalysis is proposed which looks at one or two mathematical structures as isoreflective or autoreflective pair as a mirror-like reflection of each other that expresses the magnitude of their symmetry or identity and similarity. Optinalysis is not a method for deciding that two finite graphs are isomorphic or automorphic, but extends to express the degree to which they are symmetrical, similar, and identical to each

Page 1 of 30 
Optinalysis

other. Optinalysis is well proven to be a bijective function on multiplicative inverses of its isoreflective pair of points.

\section{Preliminary definitions and theorems}

Definition I. Injections, surjections, and bijections of functions between sets, subsets [3].

These are words that describe certain functions $f: A \rightarrow B \rightarrow \mathrm{B}$ from one set to another.

An injection, also called a one-to-one function is a function that maps distinct elements to distinct elements, that is, if $x \neq y$, then $f(x) \neq f(y)$. Equivalently, if $f(x)=f(y)$ then, $x=y$.

$A$ surjection also called an onto function is one that includes all of $B$ in its image, that is, if $y \in B$, then there is an $x \in A$ such that $f(x)=y$.

$A$ bijection, also called a one-to-one and onto correspondence, is a function that is simultaneously injective and surjective. Another way to describe a bijection $f: A \rightarrow B$ is to say that there is an inverse function $g: B \rightarrow A$ so that the composition $g$ of $: A \rightarrow A$ is the identity function on $A$ while $f$ o $g: B \rightarrow B$ is the identity function on $B$. The usual notation for the function inverse to $f$ is $f^{-1}$.

If $f$ and $g$ are inverse to each other, that is, if $g$ is the inverse of $f, g=f^{-1}$, then $f$ is the inverse of $g, f=g^{-1}$ Thus, $\left(f^{-1}\right)^{-1}=f$.

An important property of bijections is that you can convert equations involving $f$ to equations involving $f^{-1}$ :

$$
f(x)=y \text { if and only if } x=f^{-1}(y) .
$$

Definition II. Isometry (or congruence or congruent transformation) is a distance-preserving transformation between metric spaces, usually assumed to be bijective. Let $A$ and $B$ be metric space with metrics $d_{A}$ and $d_{B}$. A map $f: A \rightarrow B$ is called an isometry or distance preserving if for any $a, b \in A$ one has

$$
d_{B}\left(f(a), f(b)=d_{A}(a, b)\right.
$$

[1], [3].

Definition III. Isomorphism is a vertex bijection that preserves the mathematical structures (e.g, vertices, edges, non-edges, and connections) between two spaces and graphs that can be reversed by inverse mapping. Two mathematical structures $A$ and $B$ are isomorphic if they have the same structure, but their elements may be different [2], [3].

$$
\begin{gathered}
f: A \rightarrow B \\
A \cong B
\end{gathered}
$$

Definition $I V$. Automorphism is an isomorphism from a mathematical object to itself. It is, in some sense; define as the symmetry of the object, and a way of mapping the object to itself while preserving all of its mathematical structure (e.g vertices, edges, non-edges, and connections) [2], [3].

$$
\begin{gathered}
f: A \rightarrow \operatorname{Aut}\left(A^{\prime}\right) \\
A \cong A^{\prime}
\end{gathered}
$$


Optinalysis

Definition $V$. Scale can be defined as the system of marks at fixed intervals, which define the relationship between the units being used and their representation on the graph.

Theorem I. An isometry maps

(i) straight lines to straight lines;

(ii) segments to congruent segments;

(iii) triangles to congruent triangles;

(iv) angles to congruent angles.

Theorem II. Any isometry of the plane is a composition of at most three reflections.

Theorem III. A symmetry about a point is an isometry.

\section{Definitions and Proposition of Optinalysis}

\subsection{Definition of Optinalysis}

\section{Definition 1:}

Optinalysis is a function that autoreflectively or isoreflectively compares the symmetry, similarity, and identity between two mathematical structures as a mirror-like (optic-like) reflection of each other about a symmetrical line or mid-point. In other words, it is a function that compares isoreflective or autoreflective pairs of mathematical structures.

\section{Definition 2:}

Optinalysis is a function that is comprised of an assigned optical (mirror) scale (R) that bijectively remaps $(\rightarrow)$ isoreflective or autoreflective pair of mathematical structures. Figure 1 illustrates how isoreflective pairs of points are mapped and also re-mapped by an optical scale.

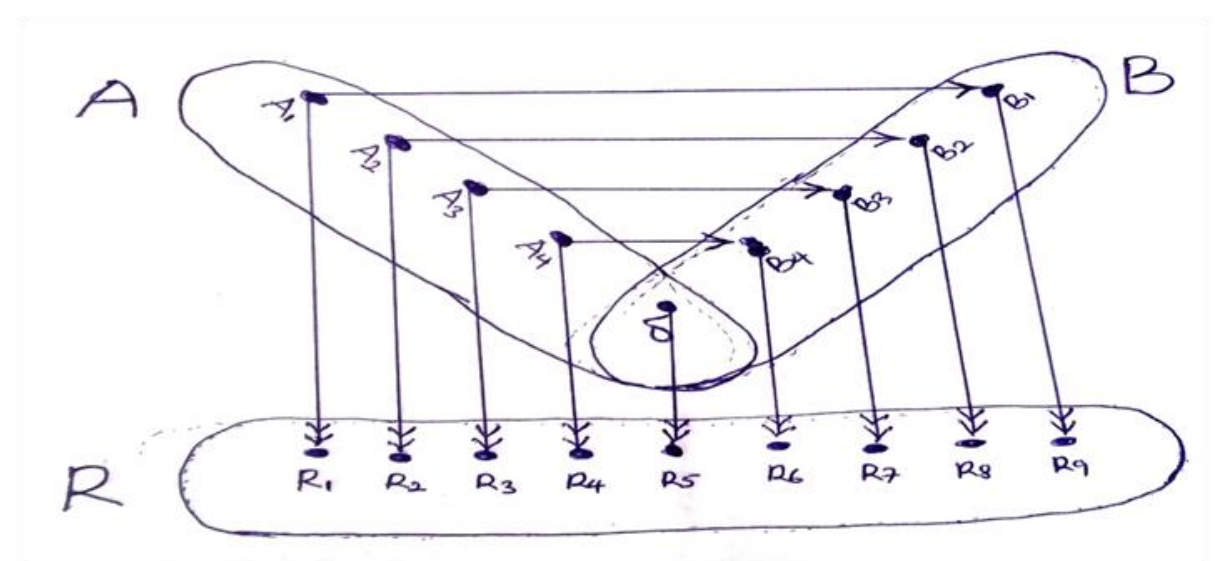

Figure I: Mapping between isoreflective pair of points and re-mapping with the optical scale. $A$ is a domain; $B$ is a co-domain of $A ; \delta$ is a mid-point or symmetrical line, and $R$ is the optical scale. The symbol $\rightarrow$ indicates a bijective mapping between the isoreflective pair around a midpoint and $\rightarrow$ indicates a bijective re-mapping by the optical scale $R$.

Optinalysis is expressed in optinalytic construction. An optinalytic construction is the mathematical representation of optinalysis between isoreflective or autoreflective pairs. 
Optinalysis

Optinalysis is defined in two broad types: shape (automorphic or intrametric) and comparative (isomorphic or intermetric) optinalysis.

\section{Definition 2.1: Autoreflective Pair}

Autoreflective pair refers to split parts of a single mathematical structure or points under reflection about a central midpoint. An autoreflectivity refers to the logical and meaningful essence or property of being autoreflective.

\section{Definition 2.2: Isoreflective Pair}

Isoreflective pair refers to two mathematical structures or points under reflection about a central midpoint. An isoreflectivity refers to the logical and meaningful essence or property of being isoreflective.

\section{Definition 2.3: Shape (Automorphic or Intrametric) Optinalysis:}

Shape or automorphic or intrametric optinalysis refers to the analysis (of symmetry) between autoreflective pairs of mathematical structure under optinalysis. It is a method of symmetry detection. Shape optinalysis is defined by its optinalytic constructions as:

$$
\begin{aligned}
& f: A \begin{array}{cc}
\delta & A^{\prime} \rightarrow R
\end{array} \\
& f: A=\left(A_{1}, A_{2}, A_{3}, \ldots, A_{n}\right) \quad \underset{m}{\delta} \quad A^{\prime}=\left(A^{\prime}{ }_{n}, \ldots, A^{\prime}{ }_{3}, A^{\prime}{ }_{2}, A^{\prime}{ }_{1}\right) \rightarrow R=\left( \pm R_{1}, \pm R_{2}, \pm R_{3}, \ldots, \pm R_{2 n+1}\right) \\
& f:\left[\begin{array}{ccc}
A=\left(A_{1}, A_{2}, A_{3}, \ldots, A_{n}\right) & \delta & A^{\prime}=\left(A_{n} \ldots, A^{\prime}{ }_{3}, A^{\prime}{ }_{2}, A^{\prime}{ }_{1}\right) \\
\Downarrow & \stackrel{\Pi}{\Downarrow} & \Downarrow \\
R=\left( \pm R_{1}, \pm R_{2}, \pm R_{3}, \ldots \pm R_{n}\right) & R_{n+1} & \left( \pm R_{n+2} \ldots, \pm R_{2 n-1}, \pm R_{2 n}, \pm R_{2 n+1}\right)
\end{array}\right]
\end{aligned}
$$

Such that: $\left(A_{1}, A_{2}, A_{3}, \ldots, A_{n}\right) \in A ;\left(A^{\prime}{ }_{1}, A^{\prime}{ }_{2}, A^{\prime}{ }_{3}, \ldots, A^{\prime}{ }_{n}\right) \in A^{\prime} ; \delta \in A \& A^{\prime} ; A, A^{\prime} \& R \in \mathbb{R}, \mathbb{R}^{\mathrm{m}} ; R_{1} \neq 0$; and $A \& B$ are autoreflective pair.

\section{Definition 2.4: Comparative (Isomorphic or Intermetric) Optinalysis:}

Comparative or isomorphic or intermetric optinalysis refers to the analysis (of similarity and identity measures) between isoreflective pair of mathematical structures under optinalysis. It is a method of similarity and identity measures. Comparative optinalysis is defined by its optinalytic constructions as:

$$
\begin{aligned}
& f: A \underset{\mathrm{m}}{\delta} \quad B \rightarrow R \\
& f: A=\left(A_{1}, A_{2}, A_{3}, \ldots, A_{n}\right) \quad \stackrel{\delta}{\mathrm{m}} \quad B=\left(B_{n}, \ldots, B_{3}, B_{2}, B_{1}\right) \rightarrow R=\left( \pm R_{1}, \pm R_{2}, \pm R_{3}, \ldots, \pm R_{2 n+1}\right) \\
& f:\left[\begin{array}{ccc}
A=\left(A_{1}, A_{2}, A_{3}, \ldots, A_{n}\right) & \delta & B=\left(B_{n}, \ldots, B_{3}, B_{2}, B_{1}\right) \\
\Downarrow & \stackrel{m}{m} & \Downarrow \\
R=\left( \pm R_{1}, \pm R_{2}, \pm R_{3}, \ldots \pm R_{n}\right) & R_{n+1} & \left( \pm R_{n+2} \ldots, \pm R_{2 n-1}, \pm R_{2 n}, \pm R_{2 n+1}\right)
\end{array}\right]
\end{aligned}
$$

Such that: $\left(A_{1}, A_{2}, A_{3}, \ldots, A_{n}\right) \in A ;\left(B_{1}, B_{2}, B_{3}, \ldots, B_{n}\right) \in B ; \delta \notin A \& B ; A, B \& R \in \mathbb{R}, \mathbb{R}^{\mathrm{m}} ; R_{1} \neq 0 ;$ and $A \& B$ are isoreflective pair. 
Optinalysis

\section{Definition 2.5: Head-to-head Reflection or Pairing}

In comparative optinalysis, a reflection (pairing) is said to be head-to-head if the lower order elements (observations) of the isoreflective pair (of two mathematical structures) are extreme away from the midpoint.

$$
A=\left(A_{1}, A_{2}, A_{3}, \ldots \ldots, A_{n}\right) \underset{\leftrightarrow}{\delta} \quad B=\left(B_{n}, \ldots \ldots, B_{3}, B_{2}, B_{1}\right)
$$

\section{Definition 2.6: Tail-to-tail Reflection or Pairing}

In comparative optinalysis, a reflection or pairing is said to be tail-to-tail if the lower order elements (observations) of the isoreflective pair (of two mathematical structures) are extreme towards the midpoint.

$$
A=\left(A_{n}, \ldots \ldots, A_{3}, A_{2}, A_{1}\right) \underset{\leftrightarrow}{\delta} \quad B=\left(B_{1}, B_{2}, B_{3}, \ldots \ldots, B_{n}\right)
$$

\section{Definition 2.7: Scalement}

A scalement, refers to the product of any member of isoreflective or autoreflective pair of a mathematical structure and its assigned optical scale.

\subsection{Proposition of Optinalysis}

\subsubsection{Proposition (Theorem) 1: Isomorphic Optinalytic}

Isoreflective pair of mathematical structures under optinalysis are similar and identical to a certain magnitude by a coefficient, called optinalytic coefficient (e.g, Kabirian coefficient, denoted as $K C$ ).

\section{Definition 4:}

Kabirian coefficient is expressed as the divisible product of the median optical scale and the sum of all members (elements) by the sum of all scalements.

\section{Prove:}

Suppose we have an optinalytic construction of isoreflective pair of mathematical structures $A$ and $B$ with an assigned optical scale $(R)$ as follows:

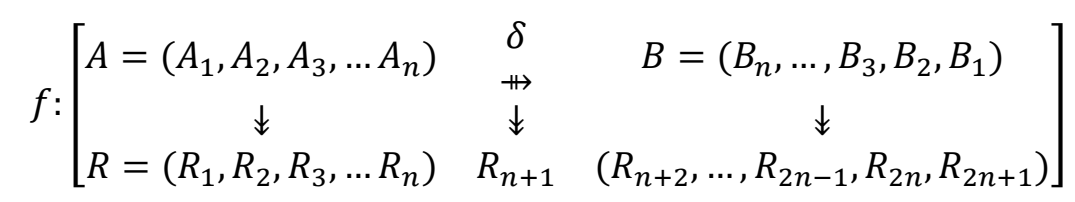

Such that: $\left(A_{1}, A_{2}, A_{3}, \ldots, A_{n}\right) \in A ;\left(B_{1}, B_{2}, B_{3}, \ldots, B_{n}\right) \in B ; \delta \notin A \& B ; A, B \& R \in \mathbb{R}, \mathbb{R}^{\mathrm{m}} ; R_{1} \neq 0$; and $A \& B$ are isoreflective pairs on a chosen pairing about a central line $(\delta)$.

Then, the Kabirian coefficient of identity and similarity between the isoreflective pair is expressed as (Equations 1):

$$
\begin{array}{r}
K C_{\text {Sim. } / I d .}(A, B)=\frac{R_{n+1}\left(A_{1}+A_{2}+A_{3}+\cdots+A_{n}+\delta+B_{n}+\cdots+B_{3}+B_{2}+B_{1}\right)}{\left(R_{1} \cdot A_{1}\right)+\left(R_{2} \cdot A_{2}\right)+\left(R_{3} \cdot A_{3}\right)+\cdots+\left(R_{n} \cdot A_{n}\right)+\left(R_{n+1} \cdot \delta\right)+} \\
\left(R_{n+2} \cdot B_{n}\right)+\cdots+\left(R_{2 n-1} \cdot B_{3}\right)+\left(R_{2 n} \cdot B_{2}\right)+\left(R_{2 n+1} \cdot B_{1}\right)
\end{array}
$$


Optinalysis

$$
\left\{\begin{aligned}
K C_{\text {Sim. } / I d .}(A, B)=1, & \text { if }|E(A)|=|E(B)| \\
K C_{\text {Sim. } / I d .}(A, B)=0, & \text { if }|E(A)|=-|E(B)| ;-|E(A)|=|E(B)| \\
0 \leq K C_{\text {Sim. } / I d .}(A, B) \leq 1, & \text { if }|E(A)|<|E(B)| \\
1 \leq K C_{\text {Sim. } / I d .}(A, B) \leq n+1, & \text { if }|E(A)|>|E(B)| \\
K C_{\text {Sim. } / I d .}(A, B) \geq n+1,<0, & \text { if }|E(A)|>E|E(B)|
\end{aligned}\right.
$$

Where $|E(A)|$ and $|E(B)|$ are the absolute optical moment of $A$ and $B$ respectively about the midpoint through a distance $D$ started from the center. It is expressed by equations (2.1) and (2.2).

$$
\begin{gathered}
f:\left[\begin{array}{ccc}
A=\left(A_{1}, A_{2}, A_{3}, \ldots A_{n}\right) & \delta & B=\left(B_{n}, \ldots, B_{3}, B_{2}, B_{1}\right) \\
\Downarrow & \stackrel{m}{*} & \Downarrow \\
D=\left(D_{n}, D_{n-1}, D_{n-2}, \ldots D_{1}\right) & D_{0} & \left(D_{1}, \ldots, D_{n-2}, D_{n-1}, D_{n}\right)
\end{array}\right] \\
|E(A)|=\left|\left(D_{n} \cdot A_{1}\right)+\left(D_{n-1} \cdot A_{2}\right)+\left(D_{n-2} \cdot A_{3}\right)+\cdots+\left(D_{1} \cdot A_{n}\right)\right|=\left|\sum_{n=1}^{n}(D . A)\right| \\
|E(B)|=\left|\left(D_{n} \cdot B_{1}\right)+\left(D_{n-1} \cdot B_{2}\right)+\left(D_{n-2} \cdot B_{3}\right)+\cdots+\left(D_{1} \cdot B_{n}\right)\right|=\left|\sum_{n=1}^{n}(D . B)\right|
\end{gathered}
$$

\section{Lemma 1.1: The bijection function and property}

Pair of isoreflective points under optinalysis are bijective (one-to-one and onto) to each other and to the central midpoint constructively and functionally.

\section{Prove:}

Supposed we have an optinalytic construction between isoreflective pair of similar mathematical structures A and B as follow:

$$
f:\left[\begin{array}{ccc}
A=\left(x^{2}, x^{5}, x^{3}\right) & \delta & B=\left(y^{3}, y^{5}, y^{2}\right) \\
\Downarrow & \stackrel{+}{ } & \Downarrow \\
R=(1,2,3) & 4 & (5,6,7)
\end{array}\right]
$$

By Kabirian-based optinalysis, each element functions as (Equations 2.1-2.7):

$$
\begin{aligned}
& x^{2}=\frac{K_{c}\left(2 x^{5}+3 x^{3}+4 \delta+5 y^{3}+6 y^{5}+7 y^{2}\right)-4\left(x^{5}+x^{3}+\delta+y^{3}+y^{5}+y^{2}\right)}{4-K_{c}} \\
& x^{5}=\frac{K_{c}\left(x^{2}+3 x^{3}+4 \delta+5 y^{3}+6 y^{5}+7 y^{2}\right)-4\left(x^{2}+x^{3}+\delta+y^{3}+y^{5}+y^{2}\right)}{4-2 K_{c}} \\
& x^{3}=\frac{K_{c}\left(x^{2}+2 x^{5}+4 \delta+5 y^{3}+6 y^{5}+7 y^{2}\right)-4\left(x^{2}+x^{5}+\delta+y^{3}+y^{5}+y^{2}\right)}{4-3 K_{c}} \\
& \delta=\frac{K_{c}\left(x^{2}+2 x^{5}+3 x^{3}+5 y^{3}+6 y^{5}+7 y^{2}\right)-4\left(x^{2}+x^{5}+x^{3}+y^{3}+y^{5}+y^{2}\right)}{4-4 K_{c}}
\end{aligned}
$$


Optinalysis

$$
\begin{aligned}
& y^{3}=\frac{K_{c}\left(x^{2}+2 x^{5}+3 x^{3}+4 \delta+6 y^{5}+7 y^{2}\right)-4\left(x^{2}+x^{5}+x^{3}+\delta+y^{5}+y^{2}\right)}{4-5 K_{c}} \\
& y^{5}=\frac{K_{c}\left(x^{2}+2 x^{5}+3 x^{3}+4 \delta+5 y^{3}+7 y^{2}\right)-4\left(x^{2}+x^{5}+x^{3}+\delta+y^{3}+y^{2}\right)}{4-6 K_{c}} \\
& y^{2}=\frac{K_{c}\left(x^{2}+2 x^{5}+3 x^{3}+4 \delta+5 y^{3}+6 y^{5}\right)-4\left(x^{2}+x^{5}+x^{3}+\delta+y^{3}+y^{5}\right)}{4-7 K_{c}}
\end{aligned}
$$

Recall the definition of bijective mapping (one-to-one and onto), such that if $x=y$, then $f(g(x))=$ $g(f(y))$. We now have nine cases that have been evaluated (see Appendix A) in this respect as follows:

Case 1:

$$
\begin{gathered}
x^{2}=y^{2} \Rightarrow \frac{K_{c}\left(7 y^{2}\right)-4\left(y^{2}\right)}{4-K_{c}}=\frac{K_{c}\left(x^{2}\right)-4\left(x^{2}\right)}{4-7 K_{c}} \\
f\left(g\left(x^{2}\right)\right)=g\left(f\left(y^{2}\right)\right) \Rightarrow \frac{7 K_{c}-4}{4-K_{c}}=\frac{K_{c}-4}{4-7 K_{c}} \Rightarrow f\left(x^{2}\right)=g^{-1}\left(y^{2}\right), \text { then } g\left(y^{2}\right)=f^{-1}\left(x^{2}\right)
\end{gathered}
$$

Case 2:

$$
\begin{gathered}
x^{5}=y^{5} \Rightarrow \frac{K_{c}\left(6 y^{5}\right)-4\left(y^{5}\right)}{4-2 K_{c}}=\frac{K_{c}\left(2 x^{5}\right)-4\left(x^{5}\right)}{4-6 K_{c}} \\
f\left(g\left(x^{5}\right)\right)=g\left(f\left(y^{5}\right)\right) \Rightarrow \frac{6 K_{c}-4}{4-2 K_{c}}=\frac{K_{c}-4}{4-6 K_{c}} \Rightarrow f\left(x^{5}\right)=g^{-1}\left(y^{5}\right), \text { then } g\left(y^{5}\right)=f^{-1}\left(x^{5}\right)
\end{gathered}
$$

Case 3:

$$
\begin{gathered}
x^{3}=y^{3} \Rightarrow \frac{K_{c}\left(5 y^{3}\right)-4\left(y^{3}\right)}{4-3 K_{c}}=\frac{K_{c}\left(3 x^{3}\right)-4\left(x^{3}\right)}{4-5 K_{c}} \\
f\left(g\left(x^{3}\right)\right)=g\left(f\left(y^{3}\right)\right) \Rightarrow \frac{5 K_{c}-4}{4-3 K_{c}}=\frac{3 K_{c}-4}{4-5 K_{c}} \Rightarrow f\left(x^{3}\right)=g^{-1}\left(y^{3}\right), \text { then } g\left(y^{3}\right)=f^{-1}\left(x^{3}\right)
\end{gathered}
$$

Case 4:

$$
\begin{gathered}
x^{2}=\delta \Rightarrow \frac{K_{c}(4 \delta)-4(\delta)}{4-K_{c}}=\frac{K_{c}\left(x^{2}\right)-4\left(x^{2}\right)}{4-4 K_{c}} \\
f\left(g\left(x^{2}\right)\right)=g(f(\delta)) \Rightarrow \frac{4 K_{c}-4}{4-K_{c}}=\frac{K_{c}-4}{4-4 K_{c}} \Rightarrow f\left(x^{2}\right)=g^{-1}(\delta), \text { then } g(\delta)=f^{-1}\left(x^{2}\right)
\end{gathered}
$$

Case 5:

$$
\begin{gathered}
x^{5}=\delta \Rightarrow \frac{K_{c}(4 \delta)-4(\delta)}{4-2 K_{c}}=\frac{K_{c}\left(2 x^{5}\right)-4\left(x^{3}\right)}{4-4 K_{c}} \\
f\left(g\left(x^{5}\right)\right)=g(f(\delta)) \Rightarrow \frac{4 K_{c}-4}{4-2 K_{c}}=\frac{2 K_{c}-4}{4-4 K_{c}} \Rightarrow f\left(x^{5}\right)=g^{-1}(\delta), \text { then } g(\delta)=f^{-1}\left(x^{5}\right)
\end{gathered}
$$


Optinalysis

Case 6:

$$
\begin{gathered}
x^{3}=\delta \Rightarrow \frac{K_{c}(4 \delta)-4(\delta+)}{4-3 K_{c}}=\frac{\left.K_{c}\left(3 x^{3}\right)-4 x^{3}\right)}{4-4 K_{c}} \\
f\left(g\left(x^{3}\right)\right)=g(f(\delta)) \Rightarrow \frac{4 K_{c}-4}{4-3 K_{c}}=\frac{\left.3 K_{c}-4\right)}{4-4 K_{c}} \Rightarrow f\left(x^{3}\right)=g^{-1}(\delta), \text { then } g(\delta)=f^{-1}\left(x^{3}\right)
\end{gathered}
$$

Case 7:

$$
\begin{gathered}
y^{3}=\delta \Rightarrow \frac{K_{c}(4 \delta)-4(\delta)}{4-5 K_{c}}=\frac{K_{c}\left(5 y^{3}\right)-4\left(y^{3}\right)}{4-4 K_{c}} \\
f\left(g\left(y^{3}\right)\right)=g(f(\delta)) \Rightarrow \frac{4 K_{c}-4}{4-5 K_{c}}=\frac{5 K_{c}-4}{4-4 K_{c}} \Rightarrow f\left(y^{3}\right)=g^{-1}(\delta), \text { then } g(\delta)=f^{-1}\left(y^{3}\right)
\end{gathered}
$$

Case 8:

$$
\begin{gathered}
y^{5}=\delta \Rightarrow \frac{K_{c}(4 \delta)-4(\delta)}{4-6 K_{c}}=\frac{K_{c}\left(6 y^{5}\right)-4\left(y^{5}\right)}{4-4 K_{c}} \\
f\left(g\left(y^{5}\right)\right)=g(f(\delta)) \Rightarrow \frac{4 K_{c}-4}{4-6 K_{c}}=\frac{6 K_{c}-4}{4-4 K_{c}} \Rightarrow f\left(y^{5}\right)=g^{-1}(\delta), \text { then } g(\delta)=f^{-1}\left(y^{5}\right)
\end{gathered}
$$

Case 9:

$$
\begin{gathered}
y^{2}=\delta \Rightarrow \frac{K_{c}(4 \delta)-4(\delta)}{4-7 K_{c}}=\frac{K_{c}\left(7 y^{2}\right)-4\left(y^{2}\right)}{4-4 K_{c}} \\
f\left(g\left(y^{2}\right)\right)=g(f(\delta)) \Rightarrow \frac{4 K_{c}-4}{4-7 K_{c}}=\frac{7 K_{c}-4}{4-4 K_{c}} \Rightarrow f\left(y^{2}\right)=g^{-1}(\delta), \text { then } g(\delta)=f^{-1}\left(y^{2}\right)
\end{gathered}
$$

Then, from all the nine cases evaluated, we say all the isoreflective pair of points are inverses of each other and to the central midpoint constructively and functionally, but the composition of the elements may or may not have to be the same structurally. Therefore, isomorphic optinalysis is a construction and function based on bijective mapping which signifies isomorphism of defined mathematical structures.

\subsubsection{Proposition (Theorem) 2: Automorphic Optinalytic}

Autoreflective pairs of mathematical structures under optinalysis are symmetrical to a certain magnitude by a coefficient, called optinalytic coefficient (e.g, Kabirian coefficient, denoted as $K C$ ).

\section{Prove:}

Suppose we have an optinalytic construction of autoreflective pair of a mathematical structure $A$ and $A^{\prime}$ with an assigned optical scale $(R)$ as follows:

$$
f:\left[\begin{array}{ccc}
A=\left(A_{1}, A_{2}, A_{3}, \ldots A_{\frac{n}{2}-1}\right) & \delta=A_{\frac{n}{2}} & A^{\prime}=\left(A_{\frac{n}{2}+1}^{\prime}, \ldots, A_{n-2}^{\prime}, A_{n-1}^{\prime}, A_{n}^{\prime}\right) \\
\Downarrow & \Downarrow & \Downarrow \\
R=\left(R_{1}, R_{2}, R_{3}, \ldots R_{\frac{n}{2}-1}\right) & R_{\frac{n}{2}} & \left(R_{\frac{n}{2}+1}, \ldots, R_{n-2}, R_{n-1}, R_{n}\right)
\end{array}\right]
$$


Optinalysis

Such that: $\left(A_{1}, A_{2}, A_{3}, \ldots, A_{\frac{n}{2}-1}\right) \in A ;\left(A_{\frac{n}{2}+1}^{\prime}, \ldots, A_{n-2}^{\prime}, A_{n-1}^{\prime}, A_{n}^{\prime}\right) \in A^{\prime} ; \delta \in A \& A^{\prime} ; A, A^{\prime}, \delta \& R \in$ $\mathbb{R}, \mathbb{R}^{\mathbb{m}} ; R_{1} \neq 0$; and $A \& A^{\prime}$ are autoreflective pair on a chosen pairing about a central line $(\delta)$.

3):

Then, the Kabirian coefficient of symmetry between the autoreflective pair is expressed as (Equations

$$
\begin{aligned}
& K C_{\text {Sym.. }}\left(A, A^{\prime}\right)=\frac{R_{\frac{n}{2}}\left(A_{1}+A_{2}+A_{3}+\cdots+A_{\frac{n}{2}-1}+A_{\frac{n}{2}}+A_{\frac{n}{2}+1}^{\prime}+\cdots+A_{n-2}^{\prime}+A_{n-1}^{\prime}+A_{n}^{\prime}\right)}{\left(R_{1} \cdot A_{1}\right)+\left(R_{2} \cdot A_{2}\right)+\left(R_{3} \cdot A_{3}\right)+\cdots+\left(R_{\frac{n}{2}-1} \cdot A_{n}\right)+\left(R_{\frac{n}{2}} . A\right)+} \\
& \left(R_{\frac{n}{2}+1} \cdot A_{n}^{\prime}\right)+\cdots+\left(R_{n-2} \cdot A^{\prime}{ }_{3}\right)+\left(R_{n-1} \cdot A_{2}^{\prime}\right)+\left(R_{n} \cdot A_{1}^{\prime}\right) \\
& \left\{\begin{aligned}
K C_{\text {Sim. } / I d .}\left(A, A^{\prime}\right)=1, & \text { if }|E(A)|=\left|E\left(A^{\prime}\right)\right| \\
K C_{\text {Sim. } / I d .}\left(A, A^{\prime}\right)=0, & \text { if }|E(A)|=-\left|E\left(A^{\prime}\right)\right| \\
0 \leq K C_{\text {Sim. } / I d .}\left(A, A^{\prime}\right) \leq 1, & \text { if }|E(A)|<\left|E\left(A^{\prime}\right)\right| \\
1 \leq K C_{\text {Sim. } / I d .}\left(A, A^{\prime}\right) \leq n+1, & \text { if }|E(A)|>\left|E\left(A^{\prime}\right)\right| \\
K C_{\text {Sim. } / I d .}\left(A, A^{\prime}\right) \geq n+1,<0, & \text { if }|E(A)|>E\left|E\left(A^{\prime}\right)\right|
\end{aligned}\right.
\end{aligned}
$$

Where $|E(A)|$ and $\left|E\left(A^{\prime}\right)\right|$ are the absolute optical moment of $A$ and $B$ respectively about the midpoint through a distance $D$ started from the centre. It is expressed by equations (4.1) and (4.2).

$$
\begin{aligned}
& f:\left[\begin{array}{ccc}
A=\left(A_{1}, A_{2}, A_{3}, \ldots, A_{\frac{n}{2}-1}\right) & \delta=A_{\frac{n}{2}} & A^{\prime}=\left(A_{\frac{n}{2}+1}^{\prime}, \ldots, A_{n-2}^{\prime}, A_{n-1}^{\prime}, A_{n}^{\prime}\right) \\
\Downarrow & \stackrel{\sharp}{\rightarrow} & \Downarrow \\
D=\left(D_{i}, D_{i-1}, D_{i-2}, \ldots, D_{1}\right) & D_{0} & \left(D_{1}, \ldots, D_{i-2}, D_{i-1}, D_{i}\right)
\end{array}\right] \\
& |E(A)|=\left|\left(D_{i} \cdot A_{1}\right)+\left(D_{i-1} \cdot A_{2}\right)+\left(D_{i-2} \cdot A_{3}\right)+\cdots+\left(D_{1} \cdot A_{\frac{n}{2}-1}\right)\right|=\left|\sum_{i=1}^{i}(D \cdot A)\right| \\
& \left|E\left(A^{\prime}\right)\right|=\left|\left(D_{i} \cdot A_{1}^{\prime}\right)+\left(D_{i-1} \cdot A_{2}^{\prime}\right)+\left(D_{i-2} \cdot A_{3}^{\prime}\right)+\cdots+\left(D_{1} \cdot A_{\frac{n}{2}-1}^{\prime}\right)\right|=\left|\sum_{i=1}^{i}\left(D \cdot A^{\prime}\right)\right|
\end{aligned}
$$

\section{Lemma 1.2: The bijection function and property}

Pair of autoreflective points under optinalysis is bijective (one-to-one and onto) to each other and to the central midpoint constructively and functionally.

\section{Prove:}

Supposed we have an optinalytic construction between autoreflective pair of symmetrical mathematical structure $A$ and $A^{\prime}$ as follow:

$$
f:\left[\begin{array}{ccc}
A=\left(x^{2}, x^{5}, x^{3}\right) & \delta & A^{\prime}=\left(x^{\prime 3}, x^{\prime 5}, x^{\prime 2}\right) \\
\Downarrow & \stackrel{\leftrightarrow}{*} & \Downarrow \\
R=(1,2,3) & 4 & (5,6,7)
\end{array}\right]
$$

By Kabirian-based optinalysis, each element functions as (Equations 5.1-5.7): 
Optinalysis

$$
\begin{aligned}
& x^{2}=\frac{K_{c}\left(2 x^{5}+3 x^{3}+4 \delta+5 x^{\prime 3}+6 x^{\prime 5}+7 x^{\prime 2}\right)-4\left(x^{5}+x^{3}+\delta+x^{\prime 3}+x^{\prime 5}+x^{\prime 2}\right)}{4-K_{c}} \\
& x^{5}=\frac{K_{c}\left(x^{2}+3 x^{3}+4 \delta+5 x^{\prime 3}+6 x^{\prime 5}+7 x^{\prime 2}\right)-4\left(x^{2}+x^{3}+\delta+x^{\prime 3}+x^{\prime 5}+x^{\prime 2}\right)}{4-2 K_{c}} \\
& x^{3}=\frac{K_{c}\left(x^{2}+2 x^{5}+4 \delta+5 x^{\prime 3}+6 x^{\prime 5}+7 x^{\prime 2}\right)-4\left(x^{2}+x^{5}+\delta+x^{\prime 3}+x^{\prime 5}+x^{\prime 2}\right)}{4-3 K_{c}} \\
& \delta=\frac{K_{c}\left(x^{2}+2 x^{5}+3 x^{3}+5 x^{\prime 3}+6 x^{\prime 5}+7 x^{\prime 2}\right)-4\left(x^{2}+x^{5}+x^{3}+x^{\prime 3}+x^{\prime 5}+x^{\prime 2}\right)}{4-4 K_{c}} \\
& x^{\prime 3}=\frac{K_{c}\left(x^{2}+2 x^{5}+3 x^{3}+4 \delta+6 x^{\prime 5}+7 x^{\prime 2}\right)-4\left(x^{2}+x^{5}+x^{3}+\delta+x^{\prime 5}+x^{\prime 2}\right)}{4-5 K_{c}} \\
& x^{\prime 5}=\frac{K_{c}\left(x^{2}+2 x^{5}+3 x^{3}+4 \delta+5 x^{\prime 3}+7 x^{\prime 2}\right)-4\left(x^{2}+x^{5}+x^{3}+\delta+x^{\prime 3}+x^{\prime 2}\right)}{4-6 K_{c}} \\
& x^{\prime 2}=\frac{K_{c}\left(x^{2}+2 x^{5}+3 x^{3}+4 \delta+5 x^{\prime 3}+6 x^{\prime 5}\right)-4\left(x^{2}+x^{5}+x^{3}+\delta+x^{\prime 3}+x^{\prime 5}\right)}{4-7 K_{c}}
\end{aligned}
$$

The bijection function of automorphic optinalysis is similar proven in lemma 1.1 of theorem 1 . Therefore, automorphic optinalysis is a construction and function based on bijective mapping which signifies automorphism of a defined mathematical structure.

\subsubsection{Kabirian coefficients translation (KCT) models}

It translates the two possible Kabirian bi-coefficients into a magnitude at which the isoreflective pairs are similar, identical, or symmetrical to each other within a defined bound. The defined bound is considered, in this case, as the sum of all possible fractions (equals to 1) or the sum of all possible percentages (equals to $100 \%)$.

The magnitude of the function $K C_{S y m . / S i m . / I d}$. that the calculated $P_{S y m . / S i m . / I d .}$ is isoreflective to the sum of expected $P_{\text {Sym./Sim./Id. }}$ (i.e., equals to 1 or $100 \%$ ) under zero optinalytic normalization $\delta=0$, is given by the optinalytic construction below:

$$
f:\left[\begin{array}{ccc}
P_{\text {Sym./Sim./Id }} & \delta=0 & 1 \\
\Downarrow & \stackrel{\#}{ } & \downarrow \\
R=r_{1} & \left(n r_{1}+r_{1}\right) & \left(2 n r_{1}+r_{1}\right)
\end{array}\right]
$$

Or the optinalytic construction is inversely expressed as:

$$
f:\left[\begin{array}{ccc}
1 & \delta=0 & P_{\text {Sym./Sim./Id }} \\
\Downarrow & \stackrel{\sharp}{ } & \Downarrow \\
R=r_{1} & \left(n r_{1}+r_{1}\right) & \left(2 n r_{1}+r_{1}\right)
\end{array}\right]
$$

Then, Kabirian coefficient $\left(K_{c}\right)$ is defined as: 
Optinalysis

$$
K_{c}=\frac{\left(n r_{1}+r_{1}\right)\left(P_{\text {Sym. } / \text { Sim. } / I d}+1\right)}{\left(r_{1} \cdot P_{\text {Sym. } . / S i m . / I d}\right)+\left(2 n r_{1}+r_{1}\right)}
$$

Or the Kabirian coefficient $\left(K_{c}\right)$ is inversely defined as:

$$
K_{c}=\frac{\left(n r_{1}+r_{1}\right)\left(1+P_{\text {Sym. } / \text { Sim. } / I d}\right)}{r_{1}+P_{\text {Sym./Sim./Id }}\left(2 n r_{1}+r_{1}\right)}
$$

By making $P_{\text {Sym./Sim./Id }}$ the subject of the formula, we obtain a model (Equation 6):

$$
\begin{array}{r}
P_{\text {Sym. } / \text { Sim. } / I d .}=\frac{\left(n r_{1}+r_{1}\right)-K_{c}\left(2 n r_{1}+r_{1}\right)}{K_{c}-\left(n r_{1}+r_{1}\right)}, \forall 0 \leq K_{c} \leq 1 \\
\left\{\begin{array}{rr}
0 \leq P_{\text {Sim. } / I d .}(A, B) \leq 1, & \text { if } \frac{n+1}{2 n+1} \leq K C_{\text {Sim. } / I d .}(A, B) \leq 1 \\
-1 \leq P_{\text {Sim. } / I d .}(A, B) \leq 0, & \text { if } 0 \leq K C_{\text {Sim. } / I d .}(A, B) \leq \frac{n+1}{2 n+1}
\end{array}\right.
\end{array}
$$

Or inversely as (Equation 7):

$$
\begin{gathered}
P_{\text {Sym./Sim./Id. }=} x=\frac{\left(n r_{1}+r_{1}\right)-r_{1} K_{c}}{\left(2 n r_{1}+r_{1}\right) K_{c}-\left(n r_{1}+r_{1}\right)}, \forall 1 \leq K_{c} \leq n+1 ; K_{c} \geq n+1 \& \forall K_{c} \\
\leq 0 \\
\left\{\begin{aligned}
0 & \leq P_{\text {Sim. } / I d .}(A, B) \leq 1, \quad \text { if } 1 \leq K C_{\text {Sim. } / I d .}(A, B) \leq n+1 \\
-1 & \leq P_{\text {Sim. } / I d .}(A, B) \leq 0, \quad \text { if } K C_{\text {Sim. } / I d .}(A, B) \geq n+1, \leq 0
\end{aligned}\right.
\end{gathered}
$$

\subsubsection{Asymmetry and dissimilarity}

Asymmetry $\left(P_{\text {Asym.. }}\right)$ and dissimilarity/none-identity $\left(P_{\text {Dsim./Nid. }}\right)$ between two graphical sequences under optinalysis are expressed by the equations (5) and (6). Translation of Kabirian coefficient is valid if and only if the outcomes are within the range of values -1 to 1 (or -100 to 100 of its equivalent percentage).

$$
\begin{aligned}
& \text { If } P_{\text {Sym./Sim./Id }}(A, B) \geq 0 \text {, then } P_{\text {Asym./Dsim./Nid. }}(A, B)=1-P_{\text {Sym./Sim./Id }}(A, B) \\
& \text { If } P_{\text {Sym./Sim./Id }}(A, B) \leq 0 \text {, then } P_{\text {Asym./Dsim./Nid. }}(A, B)=-1-P_{\text {Sym./Sim./Id }}(A, B)
\end{aligned}
$$

\subsubsection{Properties of Optinalysis}

i. Optinalysis is bi-coefficient and translative (i.e., forward and reverse translations). It gives two possible

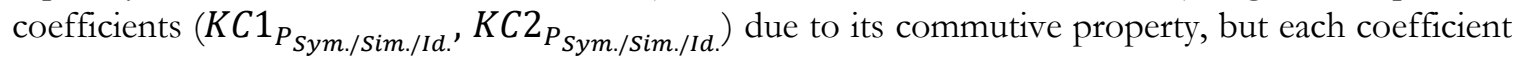
translates into the same results $\left(P_{\text {Sym./Sim./Id. }}\right.$, and $\left.P_{\text {Asym./Dsim./Nid. }}\right)$, which can be used to compute back the two coefficients.

For automorphic optinalysis

$$
\underset{K C 2_{P_{\text {Sym. }}}^{K \ldots}\left(A^{\prime}, A\right)}{K C 1_{P_{\text {Sym. }}}\left(A, A^{\prime}\right)} \rightleftharpoons P_{\text {Sym. }}\left(A, A^{\prime}\right),=P_{\text {Sym. }}\left(A^{\prime}, A\right) \rightleftharpoons P_{\text {Asym. }}\left(A, A^{\prime}\right)=P_{\text {Asym. }}\left(A^{\prime}, A\right)
$$


Optinalysis

For isomorphic optinalysis

$$
\underset{K C 1_{P_{\text {Sim./Id. }}}(A, B)}{K C 2_{P_{\text {Sim. } / I d .}}(B, A)} \rightleftharpoons P_{\text {Sim. } / I d .}(A, B),=P_{\text {Sim. } / I d .}(B, A) \rightleftharpoons P_{D \text { sim. } / \text { Nid. }}(A, B)=P_{\text {Dsim. } / \text { Nid. }}(B, A)
$$

The two possible Kabirian bi-coefficients work on different optinalytic scales.

ii. The complete symmetry, identity, or similarity between isoreflective or autoreflective pair of mathematical structures is invariant (remain the same) under transformations such as pericentral rotation (alternate reflection), central rotation (inversion), product translation, additive translation, optical scaling, and central modulation. Find the prove in Appendix B.

iii. The asymmetry or dissimilarity between isoreflective or autoreflective pair of mathematical structures is invariant (remain the same) under product translation, central rotation (inversion), and optical scaling. Find the prove in Appendix C.

iv. Under optinalytic normalization, complete symmetry, similarity, and identity (i.e., $K C=2$ ) between isoreflective pair of mathematical structures remains invariant, but asymmetry, dissimilarity, and noneidentity are normalized to a relative extent. Find the details in Appendix D.

v. The isoreflective polymorphism of mathematical structures under optinalysis, behaves on polynomial and non-polynomial models. See Appendix E for details.

\section{Discussion}

In this paper, optinalysis expressed an important paradigm for symmetry detections, similarity, and identity measures between isoreflective or autoreflective pairs of mathematical structures. The stated propositions of optinalysis and the methodological paradigm that governs it conform to the definitions and propositions of isometry, automorphism and isomorphism. The uniform intervals of the optical scale preserve an equidistant relationship between the corresponding mathematical structures. Furthermore, the optinalytic relationship between any member of isoreflective or autoreflective pair of mathematical structures is a clear bijection, and a multiplicative inverse in construction and function.

The estimates produced by optinalysis are invariant under a set of mathematical operations or transformations such as optical scaling, rotation, translation, etc. These invariance properties of optinalysis are sufficient evidence to prove its robustness for symmetry detection, similarity, and identity measurements. The moving and changing regression pattern (from the best fits of linear $\rightarrow$ exponential $\rightarrow$ polynomial $\rightarrow$ logarithmic $\rightarrow$ power) of isoreflective polymorphism of a mathematical structures under optinalysis is another interesting property observed.

\section{Conclusion}

Optinalysis is a new paradigm proposed for symmetry detections, similarity, and identity measures between isoreflective or autoreflective pairs of mathematical structures. The paradigm of optinalysis is the scale bijective re-mapping of isoreflective or autoreflective pairs. Optinalysis is characterized as invariant under transformations and its isoreflective polymorphism behaves on polynomial and non-polynomial models.

\section{$6 \quad$ Suggestion for future research}

i. The statistical goodness (robustness, unbiasedness, efficiency, and consistency) of optinalysis as an estimator for symmetry detection, similarity, and identity measures should be studied.

ii. Other modifications application of the paradigm of optinalysis should be further evaluated.

Supplementary material: The supplementary file is a customized Excel sheet for short-range tests in optinalysis. 
Optinalysis

Conflict of interest: The author declares no conflict of interest.

Funding: This research did not receive any specific grant from funding agencies in the public, commercial, or not-for-profit sectors.

\section{References}

[1] R. S. Millman and G. D. Parker, The Theory of Isometries. In: Geometry. Undergraduate Texts in Mathematics. New York, NY: Springer, 1981.

[2] O. Hatori, T. Miura, and H. Takagi, "Characterizations of isometric isomorphisms between uniform algebras via nonlinear range-preserving properties," Am. Math. Soc., vol. 134, no. 10, pp. 2923-2930, 2006.

[3] D. Joyce, "Isomorphisms: Math 130 Linear Algebra," 2015. [Online]. Available: http://math.clarku.edu/ ma130/. [Accessed: 02-May-2020]. 
Optinalysis

\section{Appendix A}

\section{The bijection function and property of optinalysis}

Pair of isoreflective points under optinalysis are bijective (one-to-one and onto) to each other and to the central midpoint constructively.

\section{Prove:}

Supposed we have an optinalytic construction between isoreflective pair of similar mathematical structures A and B as follow:

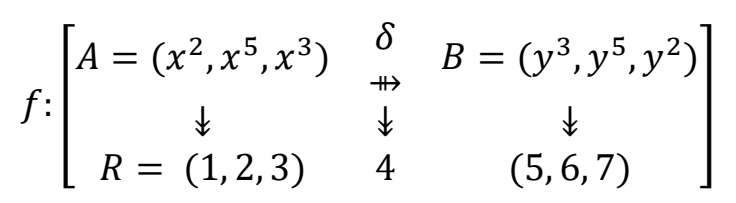

By Kabirian-based optinalysis, each element functions as (Equations A1-A7):

$$
\begin{aligned}
& x^{2}=\frac{K_{c}\left(2 x^{5}+3 x^{3}+4 \delta+5 y^{3}+6 y^{5}+7 y^{2}\right)-4\left(x^{5}+x^{3}+\delta+y^{3}+y^{5}+y^{2}\right)}{4-K_{c}} \\
& x^{5}=\frac{K_{c}\left(x^{2}+3 x^{3}+4 \delta+5 y^{3}+6 y^{5}+7 y^{2}\right)-4\left(x^{2}+x^{3}+\delta+y^{3}+y^{5}+y^{2}\right)}{4-2 K_{c}} \\
& x^{3}=\frac{K_{c}\left(x^{2}+2 x^{5}+4 \delta+5 y^{3}+6 y^{5}+7 y^{2}\right)-4\left(x^{2}+x^{5}+\delta+y^{3}+y^{5}+y^{2}\right)}{4-3 K_{c}} \\
& \delta=\frac{K_{c}\left(x^{2}+2 x^{5}+3 x^{3}+5 y^{3}+6 y^{5}+7 y^{2}\right)-4\left(x^{2}+x^{5}+x^{3}+y^{3}+y^{5}+y^{2}\right)}{4-4 K_{c}} \\
& y^{3}=\frac{K_{c}\left(x^{2}+2 x^{5}+3 x^{3}+4 \delta+6 y^{5}+7 y^{2}\right)-4\left(x^{2}+x^{5}+x^{3}+\delta+y^{5}+y^{2}\right)}{4-5 K_{c}} \\
& y^{5}=\frac{K_{c}\left(x^{2}+2 x^{5}+3 x^{3}+4 \delta+5 y^{3}+7 y^{2}\right)-4\left(x^{2}+x^{5}+x^{3}+\delta+y^{3}+y^{2}\right)}{4-6 K_{c}}
\end{aligned}
$$


Optinalysis

$$
y^{2}=\frac{K_{c}\left(x^{2}+2 x^{5}+3 x^{3}+4 \delta+5 y^{3}+6 y^{5}\right)-4\left(x^{2}+x^{5}+x^{3}+\delta+y^{3}+y^{5}\right)}{4-7 K_{c}}
$$

Recall the definition of bijective mapping (one-to-one and onto), such that if $x=y$, then $f(g(x))=g(f(y))$. We now have nine cases that have been evaluated in this respect as follows:

\section{Case A1:}

$$
\begin{aligned}
x^{2}=y^{2} \Rightarrow \frac{K_{c}\left(2 x^{5}+3 x^{3}+4 \delta+5 y^{3}+6 y^{5}+7 y^{2}\right)-4\left(x^{5}+x^{3}+\delta+y^{3}+y^{5}+y^{2}\right)}{4-K_{c}} & \\
= & \frac{K_{c}\left(x^{2}+2 x^{5}+3 x^{3}+4 \delta+5 y^{3}+6 y^{5}\right)-4\left(x^{2}+x^{5}+x^{3}+\delta+y^{3}+y^{5}\right)}{4-7 K_{c}}
\end{aligned}
$$

Divide both sides by a common factor $K_{c}\left(2 x^{5}+3 x^{3}+4 \delta+5 y^{3}+6 y^{5}\right)-4\left(x^{5}+x^{3}+\delta+y^{3}+y^{5}\right)$

Compose $f\left(x^{2}\right)$ onto $g\left(y^{2}\right)$ and vice versa

$$
x^{2}=y^{2} \Rightarrow \frac{K_{c}\left(7 y^{2}\right)-4\left(y^{2}\right)}{4-K_{c}}=\frac{K_{c}\left(x^{2}\right)-4\left(x^{2}\right)}{4-7 K_{c}}
$$

Case A2:

$$
f\left(g\left(x^{2}\right)\right)=g\left(f\left(y^{2}\right)\right) \Rightarrow \frac{7 K_{c}-4}{4-K_{c}}=\frac{K_{c}-4}{4-7 K_{c}} \Rightarrow f\left(x^{2}\right)=g^{-1}\left(y^{2}\right), \text { then } g\left(y^{2}\right)=f^{-1}\left(x^{2}\right)
$$

$$
\begin{array}{r}
x^{5}=y^{5} \Rightarrow \frac{K_{c}\left(x^{2}+3 x^{3}+4 \delta+5 y^{3}+6 y^{5}+7 y^{2}\right)-4\left(x^{2}+x^{3}+\delta+y^{3}+y^{5}+y^{2}\right)}{4-2 K_{c}} \\
=\frac{K_{c}\left(x^{2}+2 x^{5}+3 x^{3}+4 \delta+5 y^{3}+7 y^{2}\right)-4\left(x^{2}+x^{5}+x^{3}+\delta+y^{3}+y^{2}\right)}{4-6 K_{c}}
\end{array}
$$

Divide both sides by a common factor $K_{c}\left(x^{2}+3 x^{3}+4 \delta+5 y^{3}+7 y^{2}\right)-4\left(x^{2}+x^{3}+\delta+y^{3}+y^{2}\right)$

Compose $f\left(x^{5}\right)$ onto $g\left(y^{5}\right)$ and vice versa

$$
x^{5}=y^{5} \Rightarrow \frac{K_{c}\left(6 y^{5}\right)-4\left(y^{5}\right)}{4-2 K_{c}}=\frac{K_{c}\left(2 x^{5}\right)-4\left(x^{5}\right)}{4-6 K_{c}}
$$

Case A3:

$$
f\left(g\left(x^{5}\right)\right)=g\left(f\left(y^{5}\right)\right) \Rightarrow \frac{6 K_{c}-4}{4-2 K_{c}}=\frac{K_{c}-4}{4-6 K_{c}} \Rightarrow f\left(x^{5}\right)=g^{-1}\left(y^{5}\right), \text { then } g\left(y^{5}\right)=f^{-1}\left(x^{5}\right)
$$


Optinalysis

$$
\begin{aligned}
x^{3}=y^{3} \Rightarrow \frac{K_{c}\left(x^{2}+2 x^{5}+4 \delta+5 y^{3}+6 y^{5}+7 y^{2}\right)-4\left(x^{2}+x^{5}+\delta+y^{3}+y^{5}+y^{2}\right)}{4-3 K_{c}} & \\
= & \frac{K_{c}\left(x^{2}+2 x^{5}+3 x^{3}+4 \delta+6 y^{5}+7 y^{2}\right)-4\left(x^{2}+x^{5}+x^{3}+\delta+y^{5}+y^{2}\right)}{4-5 K_{c}}
\end{aligned}
$$

Divide both sides by a common factor $K_{c}\left(x^{2}+2 x^{5}+4 \delta+6 y^{5}+7 y^{2}\right)-4\left(x^{2}+x^{5}+\delta+y^{5}+y^{2}\right)$

Compose $f\left(x^{3}\right)$ onto $g\left(y^{3}\right)$ and vice versa

$$
x^{3}=y^{3} \Rightarrow \frac{K_{c}\left(5 y^{3}\right)-4\left(y^{3}\right)}{4-3 K_{c}}=\frac{K_{c}\left(3 x^{3}\right)-4\left(x^{3}\right)}{4-5 K_{c}}
$$

Case A4:

$$
f\left(g\left(x^{3}\right)\right)=g\left(f\left(y^{3}\right)\right) \Rightarrow \frac{5 K_{c}-4}{4-3 K_{c}}=\frac{3 K_{c}-4}{4-5 K_{c}} \Rightarrow f\left(x^{3}\right)=g^{-1}\left(y^{3}\right), \text { then } g\left(y^{3}\right)=f^{-1}\left(x^{3}\right)
$$

$$
\begin{aligned}
x^{2}=\delta \Rightarrow \frac{K_{c}\left(2 x^{5}+3 x^{3}+4 \delta+5 y^{3}+6 y^{5}+7 y^{2}\right)-4\left(x^{5}+x^{3}+\delta+y^{3}+y^{5}+y^{2}\right)}{4-K_{c}} & \\
= & \frac{K_{c}\left(x^{2}+2 x^{5}+3 x^{3}+5 y^{3}+6 y^{5}+7 y^{2}\right)-4\left(x^{2}+x^{5}+x^{3}+y^{3}+y^{5}+y^{2}\right)}{4-4 K_{c}}
\end{aligned}
$$

Divide both sides by a common factor $K_{c}\left(2 x^{5}+3 x^{3}+5 y^{3}+6 y^{5}+7 y^{2}\right)-4\left(x^{5}+x^{3}+y^{3}+y^{5}+y^{2}\right)$

Compose $f\left(x^{2}\right)$ onto $g(\delta)$ and vice versa

$$
x^{2}=\delta \Rightarrow \frac{K_{c}(4 \delta)-4(\delta)}{4-K_{c}}=\frac{K_{c}\left(x^{2}\right)-4\left(x^{2}\right)}{4-4 K_{c}}
$$

Case A5:

$$
f\left(g\left(x^{3}\right)\right)=g(f(\delta)) \Rightarrow \frac{4 K_{c}-4}{4-K_{c}}=\frac{K_{c}-4}{4-4 K_{c}} \Rightarrow f\left(x^{2}\right)=g^{-1}(\delta), \text { then } g(\delta)=f^{-1}\left(x^{2}\right)
$$

$$
\begin{aligned}
x^{5}=\delta \Rightarrow \frac{K_{c}\left(x^{2}+3 x^{3}+4 \delta+5 y^{3}+6 y^{5}+7 y^{2}\right)-4\left(x^{2}+x^{3}+\delta+y^{3}+y^{5}+y^{2}\right)}{4-2 K_{c}} & \\
= & \frac{K_{c}\left(x^{2}+2 x^{5}+3 x^{3}+5 y^{3}+6 y^{5}+7 y^{2}\right)-4\left(x^{2}+x^{5}+x^{3}+y^{3}+y^{5}+y^{2}\right)}{4-4 K_{c}}
\end{aligned}
$$

Divide both sides by a common factor $K_{c}\left(x^{2}+3 x^{3}+5 y^{3}+6 y^{5}+7 y^{2}\right)-4\left(x^{2}+x^{3}+y^{3}+y^{5}+y^{2}\right)$

Compose $f\left(x^{5}\right)$ onto $g(\delta)$ and vice versa

$$
x^{5}=\delta \Rightarrow \frac{K_{c}(4 \delta)-4(\delta)}{4-2 K_{c}}=\frac{K_{c}\left(2 x^{5}\right)-4\left(x^{3}\right)}{4-4 K_{c}}
$$


Optinalysis

Case A6:

$$
f\left(g\left(x^{5}\right)\right)=g(f(\delta)) \Rightarrow \frac{4 K_{c}-4}{4-2 K_{c}}=\frac{2 K_{c}-4}{4-4 K_{c}} \Rightarrow f\left(x^{5}\right)=g^{-1}(\delta), \text { then } g(\delta)=f^{-1}\left(x^{5}\right)
$$

$$
\begin{aligned}
x^{3}=\delta \Rightarrow \frac{K_{c}\left(x^{2}+2 x^{5}+4 \delta+5 y^{3}+6 y^{5}+7 y^{2}\right)-4\left(x^{2}+x^{5}+\delta+y^{3}+y^{5}+y^{2}\right)}{4-3 K_{c}} & \\
= & \frac{K_{c}\left(x^{2}+2 x^{5}+3 x^{3}+5 y^{3}+6 y^{5}+7 y^{2}\right)-4\left(x^{2}+x^{5}+x^{3}+y^{3}+y^{5}+y^{2}\right)}{4-4 K_{c}}
\end{aligned}
$$

Divide both sides by a common factor $K_{c}\left(x^{2}+2 x^{5}+5 y^{3}+6 y^{5}+7 y^{2}\right)-4\left(x^{2}+x^{5}+y^{3}+y^{5}+y^{2}\right)$

Compose $f\left(x^{3}\right)$ onto $g(\delta)$ and vice versa

$$
x^{3}=\delta \Rightarrow \frac{K_{c}(4 \delta)-4(\delta+)}{4-3 K_{c}}=\frac{\left.K_{c}\left(3 x^{3}\right)-4 x^{3}\right)}{4-4 K_{c}}
$$

\section{Case A7:}

$$
f\left(g\left(x^{3}\right)\right)=g(f(\delta)) \Rightarrow \frac{4 K_{c}-4}{4-3 K_{c}}=\frac{\left.3 K_{c}-4\right)}{4-4 K_{c}} \Rightarrow f\left(x^{3}\right)=g^{-1}(\delta), \text { then } g(\delta)=f^{-1}\left(x^{3}\right)
$$

$$
\begin{aligned}
y^{3}=\delta \Rightarrow \frac{K_{c}\left(x^{2}+2 x^{5}+3 x^{3}+4 \delta+6 y^{5}+7 y^{2}\right)-4\left(x^{2}+x^{5}+x^{3}+\delta+y^{5}+y^{2}\right)}{4-5 K_{c}} \\
=\frac{K_{c}\left(x^{2}+2 x^{5}+3 x^{3}+5 y^{3}+6 y^{5}+7 y^{2}\right)-4\left(x^{2}+x^{5}+x^{3}+y^{3}+y^{5}+y^{2}\right)}{4-4 K_{c}}
\end{aligned}
$$

Divide both sides by a common factor $K_{c}\left(x^{2}+2 x^{5}+3 x^{3}+6 y^{5}+7 y^{2}\right)-4\left(x^{2}+x^{5}+x^{3}+y^{5}+y^{2}\right)$

Compose $f\left(y^{3}\right)$ onto $g(\delta)$ and vice versa

$$
y^{3}=\delta \Rightarrow \frac{K_{c}(4 \delta)-4(\delta)}{4-5 K_{c}}=\frac{K_{c}\left(5 y^{3}\right)-4\left(y^{3}\right)}{4-4 K_{c}}
$$

Case A8:

$$
f\left(g\left(y^{3}\right)\right)=g(f(\delta)) \Rightarrow \frac{4 K_{c}-4}{4-5 K_{c}}=\frac{5 K_{c}-4}{4-4 K_{c}} \Rightarrow f\left(y^{3}\right)=g^{-1}(\delta), \text { then } g(\delta)=f^{-1}\left(y^{3}\right)
$$

$$
\begin{array}{r}
y^{5}=\delta \Rightarrow \frac{K_{c}\left(x^{2}+2 x^{5}+3 x^{3}+4 \delta+5 y^{3}+7 y^{2}\right)-4\left(x^{2}+x^{5}+x^{3}+\delta+y^{3}+y^{2}\right)}{4-6 K_{c}} \\
=\frac{K_{c}\left(x^{2}+2 x^{5}+3 x^{3}+5 y^{3}+6 y^{5}+7 y^{2}\right)-4\left(x^{2}+x^{5}+x^{3}+y^{3}+y^{5}+y^{2}\right)}{4-4 K_{c}}
\end{array}
$$

Divide both sides by a common factor $K_{c}\left(x^{2}+2 x^{5}+3 x^{3}+5 y^{3}+7 y^{2}\right)-4\left(x^{2}+x^{5}+x^{3}+y^{3}+y^{2}\right)$ 
Optinalysis

Compose $f\left(y^{5}\right)$ onto $g(\delta)$ and vice versa

$$
y^{5}=\delta \Rightarrow \frac{K_{c}(4 \delta)-4(\delta)}{4-6 K_{c}}=\frac{K_{c}\left(6 y^{5}\right)-4\left(y^{5}\right)}{4-4 K_{c}}
$$

Case A9:

$$
f\left(g\left(y^{5}\right)\right)=g(f(\delta)) \Rightarrow \frac{4 K_{c}-4}{4-6 K_{c}}=\frac{6 K_{c}-4}{4-4 K_{c}} \Rightarrow f\left(y^{5}\right)=g^{-1}(\delta), \text { then } g(\delta)=f^{-1}\left(y^{5}\right)
$$

$$
\begin{aligned}
y^{2}=\delta \Rightarrow \frac{K_{c}\left(x^{2}+2 x^{5}+3 x^{3}+4 \delta+5 y^{3}+6 y^{5}\right)-4\left(x^{2}+x^{5}+x^{3}+\delta+y^{3}+y^{5}\right)}{4-7 K_{c}} \\
=\frac{K_{c}\left(x^{2}+2 x^{5}+3 x^{3}+5 y^{3}+6 y^{5}+7 y^{2}\right)-4\left(x^{2}+x^{5}+x^{3}+y^{3}+y^{5}+y^{2}\right)}{4-4 K_{c}}
\end{aligned}
$$

Divide both sides by a common factor $K_{c}\left(x^{2}+2 x^{5}+3 x^{3}+5 y^{3}+6 y^{5}\right)-4\left(x^{2}+x^{5}+x^{3}+y^{3}+y^{5}\right)$

Compose $f\left(y^{2}\right)$ onto $g(\delta)$ and vice versa

$$
y^{2}=\delta \Rightarrow \frac{K_{c}(4 \delta)-4(\delta)}{4-7 K_{c}}=\frac{K_{c}\left(7 y^{2}\right)-4\left(y^{2}\right)}{4-4 K_{c}}
$$

$$
f\left(g\left(y^{2}\right)\right)=g(f(\delta)) \Rightarrow \frac{4 K_{c}-4}{4-7 K_{c}}=\frac{7 K_{c}-4}{4-4 K_{c}} \Rightarrow f\left(y^{2}\right)=g^{-1}(\delta), \text { then } g(\delta)=f^{-1}\left(y^{2}\right)
$$


Optinalysis

\section{Appendix B}

\section{Property B1: Optinalytic invariance under operation (I)}

A perfect symmetry or identity and similarity state between isoreflective or autoreflective pair under optinalysis remain invariant (stable) under transformations such as pericentral rotation (alternate reflection), central rotation (inversion), product translation, additive translation, optical scaling, and central modulation.

\section{Prove B1:}

Suppose we have an optinalytic construction of isoreflective pair with an assigned optical scale $(\mathrm{R}=1$, $2,3,4,5,6,7)$ as follows:

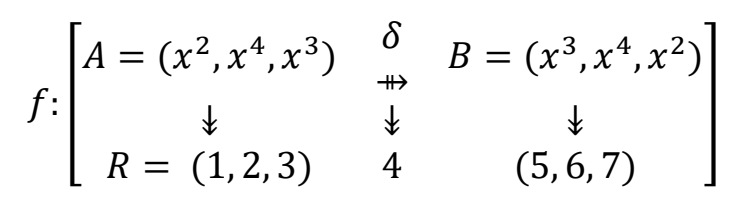

Such that $A$ and $B$ are isoreflective pairs on head-to-head reflection about a centre $(\delta)$.

Then,

$$
\begin{gathered}
K_{c}=\frac{4\left(x^{2}+x^{4}+x^{3}+\delta+x^{3}+x^{4}+x^{2}\right)}{x^{2}+2 x^{4}+3 x^{3}+4 \delta+5 x^{3}+6 x^{4}+7 x^{2}} \\
K_{c}=\frac{4 x^{2}+4 x^{4}+4 x^{3}+4 \delta+4 x^{3}+4 x^{4}+4 x^{2}}{x^{2}+2 x^{4}+3 x^{3}+4 \delta+5 x^{3}+6 x^{4}+7 x^{2}} \\
K_{c}=\frac{8 x^{2}+8 x^{3}+8 x^{4}+4 \delta}{8 x^{2}+8 x^{3}+8 x^{4}+4 \delta}=1
\end{gathered}
$$

Therefore, $A$ and $B$ are perfectly similar and identical.

\section{Prove B1.1: Invariance under additive translation}

Let the optinalytic construction of prove B1 be considered, and let ' $a$ ' be a translation factor. The optinalytic construction becomes:

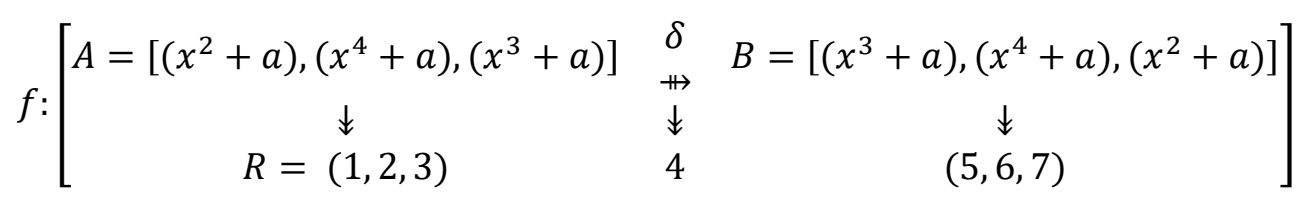

Such that $A$ and $B$ are isoreflective pairs on head-to-head reflection about a centre $(\delta)$.

Then,

$$
\begin{gathered}
K_{c}=\frac{4\left[\left(x^{2}+a\right)+\left(x^{4}+a\right)+\left(x^{3}+a\right)+\delta+\left(x^{3}+a\right)+\left(x^{4}+a\right)+\left(x^{2}+a\right)\right]}{\left(x^{2}+a\right)+2\left(x^{4}+a\right)+3\left(x^{3}+a\right)+4 \delta+5\left(x^{3}+a\right)+6\left(x^{4}+a\right)+7\left(x^{2}+a\right)} \\
K_{c}=\frac{8\left(x^{2}+a\right)+8\left(x^{4}+a\right)+8\left(x^{3}+a\right)+4 \delta}{8\left(x^{2}+a\right)+8\left(x^{4}+a\right)+8\left(x^{3}+a\right)+4 \delta}=1
\end{gathered}
$$

Therefore, $A$ and $B$ are invariant under translation. 
Optinalysis

\section{Prove B1.2: Invariance under product translation}

Let the optinalytic construction of prove B1 be considered, and let ' $a$ ' be a translation factor. The optinalytic construction becomes:

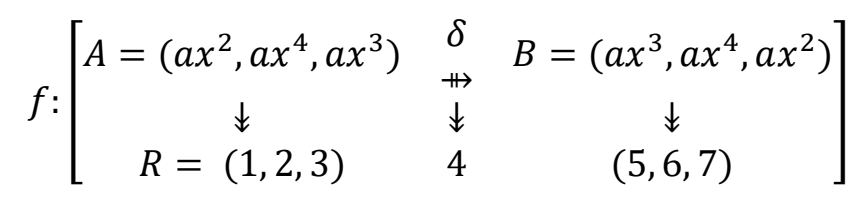

Such that $A$ and $B$ are isoreflective pairs on head-to-head reflection about a centre $(\delta)$.

Then,

$$
\begin{gathered}
K_{c}=\frac{4\left(a x^{2}+a x^{4}+a x^{3}+\delta+a x^{3}+a x^{4}+a x^{2}\right)}{a x^{2}+2 a x^{4}+3 a x^{3}+4 \delta+5 a x^{3}+6 a x^{4}+7 a x^{2}} \\
K_{c}=\frac{4 a x^{2}+4 a x^{4}+4 a x^{3}+4 \delta+4 a x^{3}+4 a x^{4}+4 a x^{2}}{a x^{2}+2 a x^{4}+3 a x^{3}+4 \delta+5 a x^{3}+6 a x^{4}+7 a x^{2}}=1 \\
K_{c}=\frac{8 a x^{2}+8 a x^{4}+8 a x^{3}+4 \delta}{8 a x^{2}+8 a x^{4}+8 a x^{3}+4 \delta}=1
\end{gathered}
$$

Therefore, $A$ and $B$ are invariant under translation.

\section{Prove B1.3: Invariance under central rotation (Inversion)}

Definition: Central rotation refers to the rotation of all the members of two mathematical structures of an isoreflective pair through $180^{\circ}$ around the central mid-point $(\boldsymbol{\delta})$. This rotation is equivalent to an inversion.

Let the optinalytic construction of prove B1 be considered, the centrally rotated structures become:

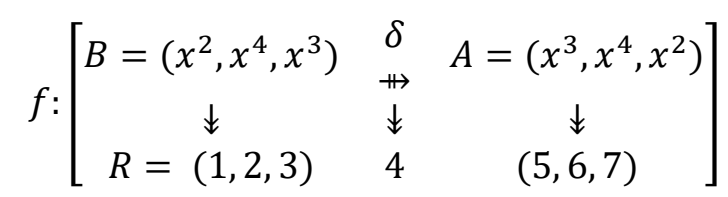

Such that $A$ and $B$ are isoreflective pairs on head-to-head reflection about a centre $(\delta)$.

Then,

$$
\begin{gathered}
K_{c}=\frac{4\left(x^{2}+x^{4}+x^{3}+\delta+x^{3}+x^{4}+x^{2}\right)}{x^{2}+2 x^{4}+3 x^{3}+4 \delta+5 x^{3}+6 x^{4}+7 x^{2}} \\
K_{c}=\frac{4 x^{2}+4 x^{4}+4 x^{3}+4 \delta+4 x^{3}+4 x^{4}+4 x^{2}}{x^{2}+2 x^{4}+3 x^{3}+4 \delta+5 x^{3}+6 x^{4}+7 x^{2}} \\
K_{c}=\frac{8 x^{2}+8 x^{3}+8 x^{4}+4 \delta}{8 x^{2}+8 x^{3}+8 x^{4}+4 \delta}=1
\end{gathered}
$$

Therefore, $A$ and $B$ are invariant under central rotation. 
Optinalysis

\section{Prove B1.4: Invariance under pericentral rotation (Alternate reflection)}

Definition: Central rotation refers to as the rotation of all the members of two mathematical structures of an isoreflective pair through $180^{\circ}$ around the pericentres (A pericentre is a mid-point of each of the two comparing mathematical structures). This structure is the same as the alternate reflection (i.e, the tail-to-tail reflection or otherwise). An alternate reflection is the alternative form of reflection between isoreflective pairs. The alternate reflection can, in some cases, be used to distinguish between two similar structures but not identical to each other.

Let the optinalytic construction of prove B1 be considered, the pericentrally rotated structures (inverses) of isoreflective pair become:

$$
f:\left[\begin{array}{ccc}
A=\left(x^{3}, x^{4}, x^{2}\right) & \delta & B=\left(x^{2}, x^{4}, x^{3}\right) \\
\Downarrow & \stackrel{\leftrightarrow}{*} & \Downarrow \\
R=(1,2,3) & 4 & (5,6,7)
\end{array}\right]
$$

Such that $A$ and $B$ are isoreflective pair on tail-to-tail (its alternate) reflection about a centre $(\delta)$.

Then,

$$
\begin{gathered}
K_{c}=\frac{4\left(x^{3}+x^{4}+x^{2}+\delta+x^{2}+x^{4}+x^{3}\right)}{x^{3}+2 x^{4}+3 x^{2}+4 \delta+5 x^{2}+6 x^{4}+7 x^{3}} \\
K_{c}=\frac{4 x^{3}+4 x^{4}+4 x^{2}+4 \delta+4 x^{2}+4 x^{4}+4 x^{3}}{x^{3}+2 x^{4}+3 x^{2}+4 \delta+5 x^{2}+6 x^{4}+7 x^{3}} \\
K_{c}=\frac{8 x^{3}+8 x^{4}+8 x^{2}+4 \delta}{8 x^{3}+8 x^{4}+8 x^{2}+4 \delta}=1
\end{gathered}
$$

Therefore, $A$ and $B$ are invariant under pericentral rotation.

\section{Prove B1.5: Invariance under optical scaling}

Let the optinalytic construction of prove $\mathrm{B} 1$ be considered, and let $R+a$ be the change in scaling patterns. The optinalytic construction becomes:

$$
f:\left[\begin{array}{ccc}
A=\left(x^{2}, x^{4}, x^{3}\right) & \delta & B=\left(x^{3}, x^{4}, x^{2}\right) \\
\Downarrow & \stackrel{+}{*} & \Downarrow \\
R=[(1+a),(2+a),(3+a)] & (4+a) & {[(5+a),(6+a),(7+a)]}
\end{array}\right]
$$

Such that $A$ and $B$ are isoreflective pairs on head-to-head reflection about a centre $(\delta)$.

Then,

$$
\begin{gathered}
K_{c}=\frac{(4+a) \times\left(x^{2}+x^{4}+x^{3}+\delta+x^{3}+x^{4}+x^{2}\right)}{x^{2}(1+a)+x^{4}(2+a)+x^{3}(3+a)+\delta(4+a)+x^{3}(5+a)+x^{4}(6+a)+x^{2}(7+a)} \\
K_{c}=\frac{4 x^{2}+4 x^{4}+4 x^{3}+4 \delta+4 x^{3}+4 x^{4}+4 x^{2}}{x^{2}+2 x^{4}+3 x^{3}+4 \delta+5 x^{3}+6 x^{4}+7 x^{2}}
\end{gathered}
$$


Optinalysis

$$
K_{c}=\frac{x^{2}(8+2 a)+x^{4}(8+2 a)+x^{3}(8+2 a)+\delta(4+a)}{x^{2}(8+2 a)+x^{4}(8+2 a)+x^{3}(8+2 a)+\delta(4+a)}=1
$$

Therefore, $A$ and $B$ are invariant under optical scaling.

\section{Prove B1.6: Invariance under central modulation}

Let the optinalytic construction of prove B1 be considered, and let $\delta \pm \beta$ be the central modulation, such that $\beta \in \mathbb{R}$. The optinalytic construction becomes:

$$
f:\left[\begin{array}{ccc}
A=\left(x^{2}, x^{4}, x^{3}\right) & \delta \pm \beta & B=\left(x^{3}, x^{4}, x^{2}\right) \\
\Downarrow & \stackrel{+}{*} & \Downarrow \\
R=(1,2,3) & 4 & (5,6,7)
\end{array}\right]
$$

Such that $A$ and $B$ are isoreflective pair on head-to-head reflection about a centre $(\delta)$.

Then,

$$
\begin{gathered}
K_{c}=\frac{4\left(x^{2}+x^{4}+x^{3}+(\delta \pm \beta) x^{3}+x^{4}+x^{2}\right)}{x^{2}+2 x^{4}+3 x^{3}+4(\boldsymbol{\delta} \pm \boldsymbol{\beta})+5 x^{3}+6 x^{4}+7 x^{2}} \\
K_{c}=\frac{4 x^{2}+4 x^{4}+4 x^{3}+4(\delta \pm \beta)+4 x^{3}+4 x^{4}+4 x^{2}}{x^{2}+2 x^{4}+3 x^{3}+4(\delta \pm \beta)+5 x^{3}+6 x^{4}+7 x^{2}} \\
K_{c}=\frac{8 x^{2}+8 x^{3}+8 x^{4}+4(\delta \pm \beta)}{8 x^{2}+8 x^{3}+8 x^{4}+4(\delta \pm \beta)}=1
\end{gathered}
$$

Therefore, $A$ and $B$ are invariant under central modulation. 
Optinalysis

\section{Appendix C}

\section{Property C1: Optinalytic invariance under operations (II)}

Asymmetrical or dissimilar state between isoreflective or autoreflective pair under optinalysis remains invariant (the same) under product translation, central rotation (inversion), and optical scaling.

\section{Prove C1:}

Suppose we have an optinalytic construction of isoreflective pair with an assigned optical scale $(\mathrm{R}=$ $1,-2,-3,-4,-5,-6,-7)$ as follows:

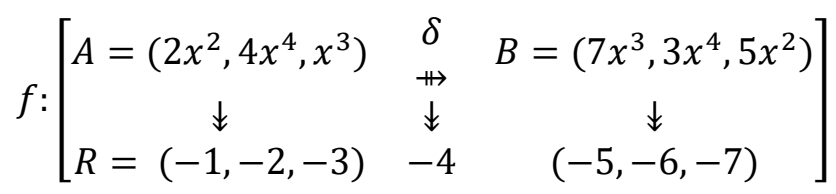

Such that $A$ and $B$ are isoreflective pair on head-to-head reflection about a centre $(\delta)$.

Then,

$$
\begin{gathered}
K_{c}=\frac{-4\left(2 x^{2}+4 x^{4}+x^{3}+\delta+7 x^{3}+3 x^{4}+5 x^{2}\right)}{-2 x^{2}-8 x^{4}-3 x^{3}-4 \delta-35 x^{3}-18 x^{4}-35 x^{2}} \\
K_{c}=\frac{-8 x^{2}-16 x^{4}-4 x^{3}-4 \delta-28 x^{3}-12 x^{4}-20 x^{2}}{-2 x^{2}-8 x^{4}-3 x^{3}-4 \delta-35 x^{3}-18 x^{4}-35 x^{2}} \\
K_{c}=\frac{\left(-28 x^{2}-28 x^{4}-32 x^{3}\right)-4 \delta}{\left(37 x^{2}-26 x^{4}-38 x^{3}\right)-4 \delta} \\
K_{c}=\frac{-88-4 \delta}{-101-4 \delta} \neq 1
\end{gathered}
$$

Therefore, $A$ and $B$ are dissimilar (asymmetrical).

\section{Prove C1.1: Invariance under product translation}

Let the optinalytic construction of prove $\mathrm{C} 1$ be considered, and let ' $a$ ' be a translation factor. The optinalytic construction becomes:

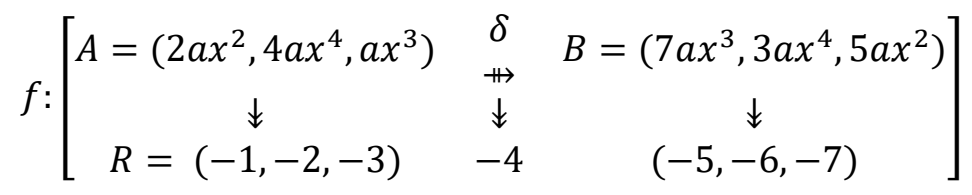

Such that $A$ and $B$ are isoreflective pair on head-to-head reflection about a centre $(\delta)$.

Then,

$$
\begin{gathered}
K_{c}=\frac{-4\left(2 a x^{2}+4 a x^{4}+a x^{3}+\delta+7 a x^{3}+3 a x^{4}+5 a x^{2}\right)}{-2 x^{2}-8 x^{4}-3 x^{3}-4 \delta-35 x^{3}-18 x^{4}-35 x^{2}} \\
K_{c}=\frac{-8 a x^{2}-16 a x^{4}-4 a x^{3}-4 \delta-28 a x^{3}-12 a x^{4}-20 a x^{2}}{-2 a x^{2}-8 a x^{4}-3 a x^{3}-4 \delta-35 a x^{3}-18 a x^{4}-35 a x^{2}}
\end{gathered}
$$


Optinalysis

$$
\begin{gathered}
K_{c}=\frac{\left(-28 a x^{2}-28 a x^{4}-32 a x^{3}\right)-4 \delta}{\left(37 a x^{2}-26 a x^{4}-38 a x^{3}\right)-4 \delta} \\
K_{c}=\frac{-88-4 \delta}{-101-4 \delta} \neq 1
\end{gathered}
$$

Therefore, $A$ and $B$ are invariant under product translation.

\section{Prove C1.2: Invariance under central rotation (Inversion)}

Let the optinalytic construction of prove $\mathrm{C} 1$ be considered, the centrally rotated structures of sequences of isometric isomorphs become:

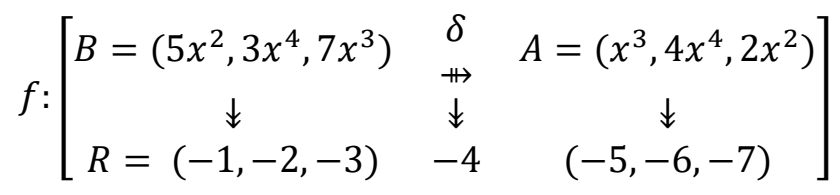

Such that $A$ and $B$ are isoreflective pair on head-to-head reflection about a centre $(\delta)$.

Then,

$$
\begin{gathered}
K_{c}=\frac{-4\left(5 x^{2}+3 x^{4}+7 x^{3}+\delta+x^{3}+4 x^{4}+2 x^{2}\right)}{-5 x^{2}-6 x^{4}-21 x^{3}-4 \delta-5 x^{3}-24 x^{4}-14 x^{2}} \\
K_{c}=\frac{-20 x^{2}-12 x^{4}-28 x^{3}-4 \delta-4 x^{3}-16 x^{4}-8 x^{2}}{-5 x^{2}-6 x^{4}-21 x^{3}-4 \delta-5 x^{3}-24 x^{4}-14 x^{2}} \\
K_{c}=\frac{\left(-28 x^{3}-28 x^{4}-32 x^{2}\right)-4 \delta}{\left(-19 x^{3}-30 x^{4}-26 x^{2}\right)-4 \delta} \\
K_{c}=\frac{-88-4 \delta}{-75-4 \delta} \neq 1
\end{gathered}
$$

Therefore, $A$ and $B$ are invariant under product translation.

\section{Prove C1.3: Invariance under optical scaling}

Let the optinalytic construction of prove $\mathrm{C} 1$ be considered, and let $(-R-a)$ be the change in scaling patterns. The optinalytic construction becomes:

$$
f:\left[\begin{array}{ccc}
A=\left(2 x^{2}, 4 x^{4}, x^{3}\right) & \delta & B=\left(7 x^{3}, 3 x^{4}, 5 x^{2}\right) \\
\Downarrow & \stackrel{+}{\sharp} & \Downarrow \\
R=[(-1-a),(-2-a),(-3-a)] & (-4-a) & {[(-5-a),(-6-a),(-7-a)]}
\end{array}\right]
$$

Such that $A$ and $B$ are isoreflective pairs on head-to-head reflection about a centre $(\delta)$.

Then,

$$
\begin{gathered}
K_{c}=\frac{(-4-a) \times\left(2 x^{2}+4 x^{4}+x^{3}+\delta+7 x^{3}+3 x^{4}+5 x^{2}\right)}{2 x^{2}(-1-a)+4 x^{4}(-2-a)+x^{3}(-3-a)+\delta(-4-a)+} \\
7 x^{3}(-5-a)+3 x^{4}(-6-a)+5 x^{2}(-7-a)
\end{gathered}
$$


Optinalysis

$$
\begin{gathered}
\left(-8 x^{2}-2 a x^{2}\right)+\left(-16 x^{4}-4 a x^{4}\right)+\left(-4 x^{3}-a x^{3}\right)+(-4 \delta-a \delta)+ \\
K_{c}=\frac{\left(-28 x^{3}-7 a x^{3}\right)+\left(-12 x^{4}-3 a x^{4}\right)+\left(-20 x^{2}-5 a x^{2}\right)}{\left(-2 x^{2}-2 a x^{2}\right)+\left(-8 x^{4}-4 a x^{4}\right)+\left(-3 x^{3}-a x^{3}\right)+(-4 \delta-a \delta)+} \\
\left(-35 x^{3}-7 a x^{3}\right)+\left(-18 x^{4}-3 a x^{4}\right)+\left(-35 x^{2}-5 a x^{2}\right) \\
K_{c}=\frac{\left(-28 x^{2}-7 a x^{2}\right)+\left(-28 x^{4}-7 a x^{2}\right)+\left(-32 x^{3}-7 a x^{2}\right)+(-4 \delta-a \delta)}{\left(37 x^{2}-7 a x^{2}\right)+\left(-26 x^{4}-7 a x^{4}\right)+\left(-38 x^{3}-7 a x^{3}\right)+(-4 \delta-a \delta)} \\
K_{c}=\frac{-88-(-4 \delta-a \delta)}{-101-(-4 \delta-a \delta)} \neq 1
\end{gathered}
$$

Therefore, $A$ and $B$ are invariant under optical scaling. 
Optinalysis

\section{Appendix D}

\section{Property D1: Optinalytic normalization}

An asymmetrical or dissimilar state between isoreflective or autoreflective pair of given mathematical structures under optinalysis, can be transformed near-symmetrical or similar states by central modulation. A central modulation refers to the deliberate increase or decrease in quantity at the central mid-point $(\delta)$. The quantity affected is called the normalization unit or value, $\beta$.

\section{Prove D1:}

Suppose we have an optinalytic construction as follows:

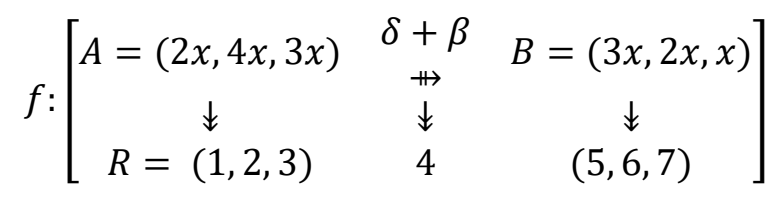

Such that $A$ and $B$ are isoreflective pair on head-to-head reflection about a centre $(\delta)$.

Then,

$$
\begin{gathered}
K_{c}=\frac{4(2 x+4 x+3 x+(\delta+\beta)+3 x+2 x+x)}{2 x+8 x+9 x+4(\delta+\beta)+15 x+12 x+7 x} \\
K_{c}=\frac{8 x+16 x+12 x+4(\delta+\beta)+12 x+8 x+4 x}{2 x+8 x+9 x+4(\delta+\beta)+15 x+12 x+7 x} \\
K_{c}=\frac{60 x+4(\delta+\beta)}{53 x+4(\delta+\beta)}
\end{gathered}
$$

Thus,

$$
K_{c}=\frac{60 x}{53 x}+\frac{4(\delta+\beta)}{4(\delta+\beta)} \neq 1
$$

Therefore, $A$ and $B$ are not perfectly symmetrical, similar, and identical.

Let $\beta= \pm 1000$ such that $\delta \pm \beta= \pm 1000$

$$
\begin{gathered}
K_{c}=\frac{60 x}{53 x}+\frac{4 \times( \pm 1000)}{4 \times( \pm 1000)} \\
K_{c}=\frac{ \pm 4060 x}{ \pm 4053 x} \cong 1
\end{gathered}
$$

Therefore, $A$ and $B$ are normalized similar or symmetrical. 
Optinalysis

\section{Appendix E}

\section{Property E1: Deterministic polynomiality and non-polynomiality of optinalysis}

The isoreflective or autoreflective polymorphism of a mathematical structure under optinalysis, behaves on polynomial and non-polynomial models.

\section{Prove E1:}

Definition: Single vertex isoreflective polymorphism: is an isomorphism as a result of single vertex variation.

Suppose we have a graph $A_{(1-N d)}$ and its isomorphic variants $B_{(1-N d)}^{g}$ from a named space. We can generate the isomorphic variants by additive paranodic skewization with a skewization value $\geq t \infty$ over some generations.

$A_{(1-N d)}=\left(y_{1}, y_{2}, y_{3}, y_{4}, y_{5}, y_{6}, \ldots \ldots y_{N d}\right)$

The single vertex isomorphic variants are:

${ }^{\text {st }}$ ranked generation $=B_{(1-N d)}^{1}=\left(y_{1}+\boldsymbol{t}, y_{2}, y_{3}, y_{4}, y_{5}, y_{6}, \ldots \ldots y_{N d}\right)$

$2^{\text {nd }}$ ranked generation $=B_{(1-N d)}^{2}=\left(y_{1}, y_{2}+\boldsymbol{t}, y_{3}, y_{4}, y_{5}, y_{6}, \ldots \ldots y_{N d}\right)$

$3^{\text {rd }}$ ranked generation $=B_{(1-N d)}^{3}=\left(y_{1}, y_{2}, y_{3}+\boldsymbol{t}, y_{4}, y_{5}, y_{6}, \ldots \ldots y_{N d}\right)$

$4^{\text {th }}$ ranked generation $=B_{(1-N d)}^{4}=\left(y_{1}, y_{2}, y_{3}, y_{4}+\boldsymbol{t}, y_{5}, y_{6}, \ldots \ldots y_{N d}\right)$

$5^{\text {th }}$ ranked generation $=B_{(1-N d)}^{5}=\left(y_{1}, y_{2}, y_{3}, y_{4}, y_{5}+\boldsymbol{t}, y_{6}, \ldots \ldots y_{N d}\right)$

$6^{\text {th }}$ ranked generation $=B_{(1-N d)}^{6}=\left(y_{1}, y_{2}, y_{3}, y_{4}, y_{5}, y_{6}+\boldsymbol{t}, \ldots \ldots y_{N d}\right)$

Alternatively, the sequence of the generations can be seen in this way:

$6^{\text {th }}$ ranked generation $=B_{(1-N d)}^{1}=\left(y_{1}+\boldsymbol{t}, y_{2}, y_{3}, y_{4}, y_{5}, y_{6}, \ldots \ldots y_{N d}\right)$

$5^{\text {th }}$ ranked generation $=B_{(1-N d)}^{2}=\left(y_{1}, y_{2}+\boldsymbol{t}, y_{3}, y_{4}, y_{5}, y_{6}, \ldots \ldots y_{N d}\right)$

$4^{\text {th }}$ ranked generation $=B_{(1-N d)}^{3}=\left(y_{1}, y_{2}, y_{3}+\boldsymbol{t}, y_{4}, y_{5}, y_{6}, \ldots \ldots y_{N d}\right)$

$3^{\text {rd }}$ ranked generation $=B_{(1-N d)}^{4}=\left(y_{1}, y_{2}, y_{3}, y_{4}+\boldsymbol{t}, y_{5}, y_{6}, \ldots \ldots y_{N d}\right)$

$2^{\text {nd }}$ ranked generation $=B_{(1-N d)}^{5}=\left(y_{1}, y_{2}, y_{3}, y_{4}, y_{5}+\boldsymbol{t}, y_{6}, \ldots \ldots y_{N d}\right)$

$1^{\text {st }}$ ranked generation $=B_{(1-N d)}^{6}=\left(y_{1}, y_{2}, y_{3}, y_{4}, y_{5}, y_{6}+\boldsymbol{t}, \ldots \ldots y_{N d}\right)$

Where the subscript $g$ in $B_{(1-N d)}^{g}$ indicate the series of generations of isomorphic point variations.

We may have an optinalytic construction as follows:

$$
f: A_{(1-N d)} \begin{gathered}
\delta=0 \\
\mathrm{~m}
\end{gathered} \quad B_{(N d-1)}^{g} \rightarrow R
$$


Optinalysis

Or in a pericentrally rotated operation (the alternate reflection) as

$$
f: A_{(N d-1)} \begin{gathered}
\delta=0 \\
\#
\end{gathered} \quad B_{(1-N d)}^{g} \rightarrow R
$$

And the inverse (centrally rotated operation) operation becomes:

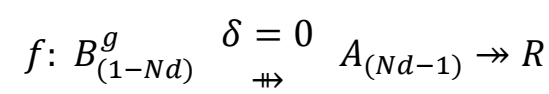

Or in a pericentrally rotated operation (the alternate reflection) as

$$
f: B_{(N d-1)}^{g} \stackrel{\delta=0}{\rightarrow} \quad A_{(1-N d)} \rightarrow R
$$

Then, the Kabirian coefficient between the graph $A_{(1-N d)}^{1}$ and its isomorphs $B_{(1-N d)}^{g}$ are obtained as $Q$ and $S$ as valid translations (in percentage or probability) of $Q$ on C-P distributions.

Let $G$ be a set of positive integers ranks the isomorphic generations (from the first to the last) of isomorphs established (for instance, $B_{(1-N d)}^{1}=1, B_{(1-N d)}^{2}=2, B_{(1-N d)}^{3}=3$, etc).

By plotting regression graphs of $G$ against $Q$, for each skewization value $t$, observe a formation and changing regression patterns (from the best fits of linear $\rightarrow$ exponential $\rightarrow$ polynomial $\rightarrow$ logarithmic $\rightarrow$ power) as a skewization value $t$ approaches a certain interval (See Appendix E).

By plotting regression graphs of $G$ against $S$, for each skewization value $t$, observe a formation and changing polynomial regression patterns as a skewization value $t$ approaches a certain interval (See Appendix E).

\section{Example Problem to Test Property E1}

Suppose we have a graph $A$ with a nodality of 20 vertices, and its single vertex isomorphs $B_{(1-20)}^{g}$ were generated using an additive paranodic skewization approach, with a skewization value $t=$ $10,25,50,10^{2}, \ldots \ldots 10^{8}$. The Kabirian coefficients and its valid translations were modeled in regressions graphically against the isomorphic generations rank, for each skewization value $(t)$.

$$
\begin{aligned}
& A_{(1-20)}=(0.73,0,15,-62,53,10,67,34,76,-34,0,35,11,20,-34,0,-26,39,0.57,-31) . \\
& B_{(1-20)}^{1} \text { or } B_{(1-20)}^{20}=(\mathbf{0 . 7 3}+\boldsymbol{t}, 0,15,-62,53,10,67,34,76,-34,0,35,11,20,-34,0,-26,39,0.57,-31) \\
& B_{(1-20)}^{2} \text { or } B_{(1-20)}^{19}=(0.73, \mathbf{0}+\boldsymbol{t}, 15,-62,53,10,67,34,76,-34,0,35,11,20,-34,0,-26,39,0.57,-31) \\
& A_{(1-20)}^{19} \text { or } B_{(1-20)}^{2}=(0.73,0,15,-62,53,10,67,34,76,-34,0,35,11,20,-34,0,-26,39, \mathbf{0 . 5 7}+\boldsymbol{t},-31) \\
& A_{(1-20)}^{20} \text { or } B_{(1-20)}^{1}=(0.73,0,15,-62,53,10,67,34,76,-34,0,35,11,20,-34,0,-26,39,0.57,-31+\boldsymbol{t})
\end{aligned}
$$

Let the optinalytic construction be defined as:

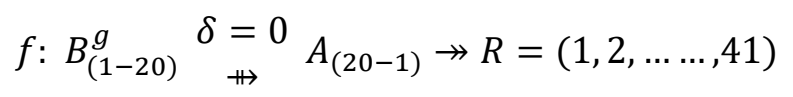

Inversely, let the optinalytic construction be defined as: 
Optinalysis

$$
f: A_{(1-20)} \stackrel{\delta}{=} \underset{\rightarrow}{\rightarrow} B_{(20-1)}^{g} \rightarrow R=(1,2, \ldots \ldots, 41)
$$

The optinalysis of these datasets, subsets was carried out using customized Excel sheets presented in the supplementary material attached to this article.

\section{Outcomes of the Exampled Problem to Test Property E1}

The Kabirian coefficient between the graph $A_{(1-N d)}^{1}$ and its isomorphs $B_{(1-N d)}^{g}$ are obtained as $Q$ and $S$ as valid translations (in percentage or probability) of $\mathcal{Q}$ on C-P distributions.

Let $G$ be a set of positive integers ranks the isomorphic generations (from the first to the last) of isomorphs established (for instance, $B_{(1-N d)}^{1}=1, B_{(1-N d)}^{2}=2, B_{(1-N d)}^{3}=3$, etc).

By plotting regression graphs of $G$ against $Q$, for each skewization value $t$, we observe moving and changing regression patterns (from the best fits of linear $\rightarrow$ exponential $\rightarrow$ polynomial, shifts to logarithmic, and finally stagnates continuously at power) as a skewization value $t$ approaches a certain interval (See Fig. E1).

However, by plotting regression graphs of $G$ against $Q$ for each skewization value $t$, of the inverse cases, we observe moving and changing regression patterns (from the best fits of linear $\rightarrow$ exponential $\rightarrow$ simple polynomial, and finally stagnates continuously at complex polynomial) as a skewization value $t$ approaches a certain interval (See Fig. E2).

Finally, by plotting regression graphs of $G$ against $S$, for each skewization value $t$, we observe moving and changing regression patterns (the best fits of from linear $\rightarrow$ exponential $\rightarrow$ simple polynomial, and finally stagnates continuously at complex polynomials) as a skewization value $t$ approaches a certain interval (See Fig. E3). Likewise, the explanations are the same for the inverse cases.

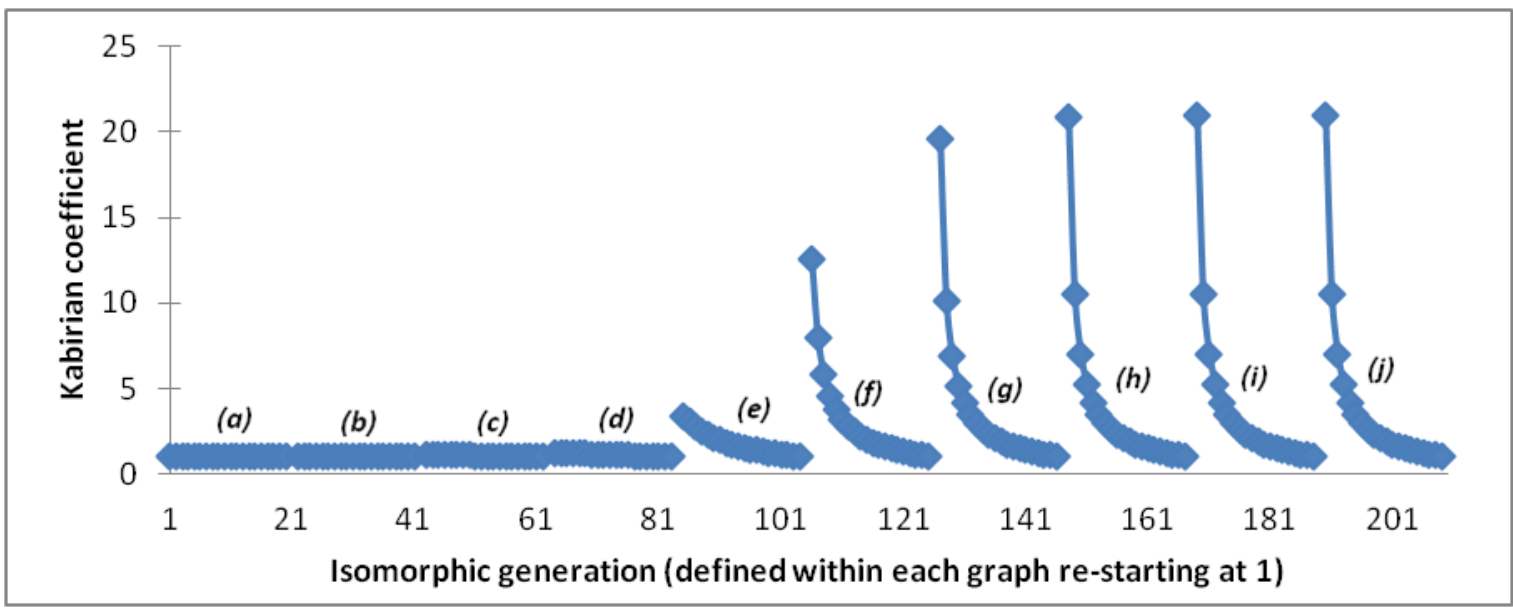

Figure E1: Regression models of the relationship between the generations rank of isomorphs and the resultant Kabirian coefficient of optinalysis. (a) $-(j)$ are the regression models at skewization value $t=$ $10,25,50,10^{2}, \ldots \ldots 10^{8}$ respectively. (a)-(e) are linear $\rightarrow$ exponential $\rightarrow$ polynomial, $(e)$ is logarithmic, and (f)-(j) are power models.

Note: In this problem, the trending $K_{c}>1$ stagnates at a magnitude equal to the median optical scale. 
Optinalysis

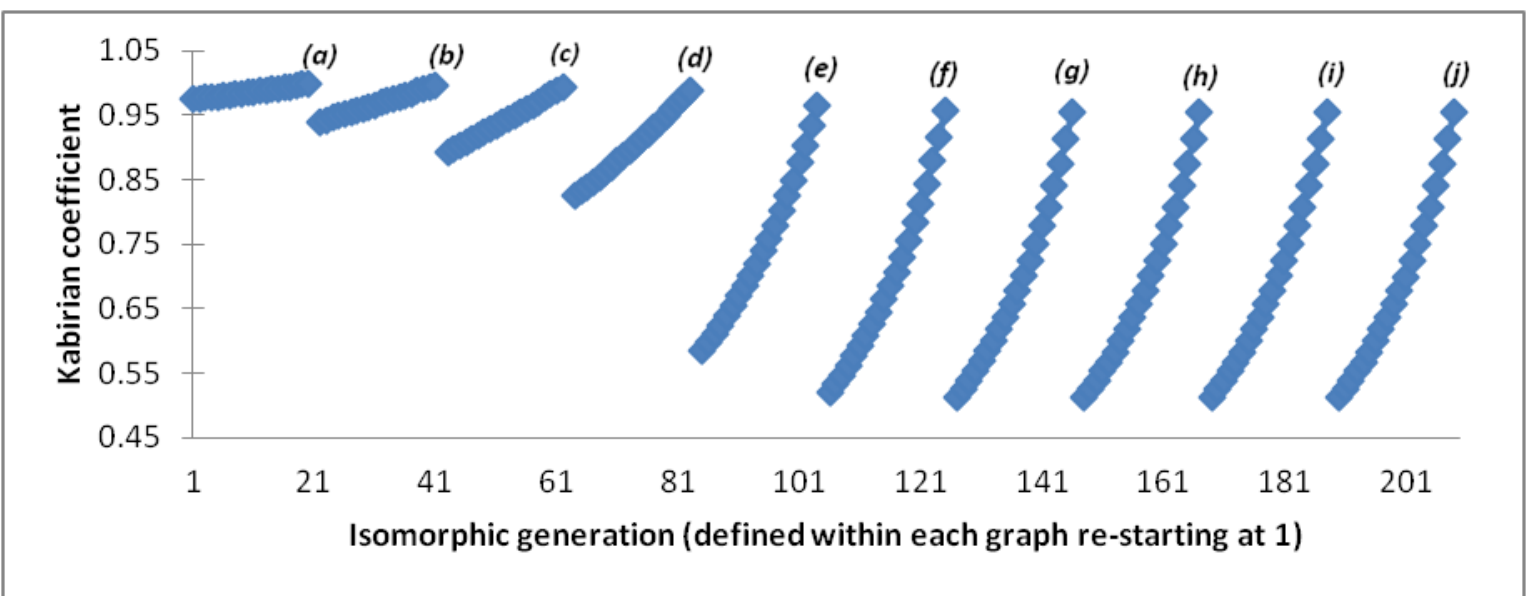

Figure E2: Regression models of the relationship between the generations rank of isomorphs and the resultant Kabirian coefficient of optinalysis of the inverse cases. (a)-(j) are the regression models at skewization value $t=10,25,50,10^{2}, \ldots \ldots 10^{8}$ respectively. (a)-(d) are linear $\rightarrow$ exponential $\rightarrow$ simple polynomial, $(e)-(j)$ are complex polynomial models.

Note: In this problem, the trending $K_{c}<1$ stagnates at a magnitude close to $\frac{1}{2}$.

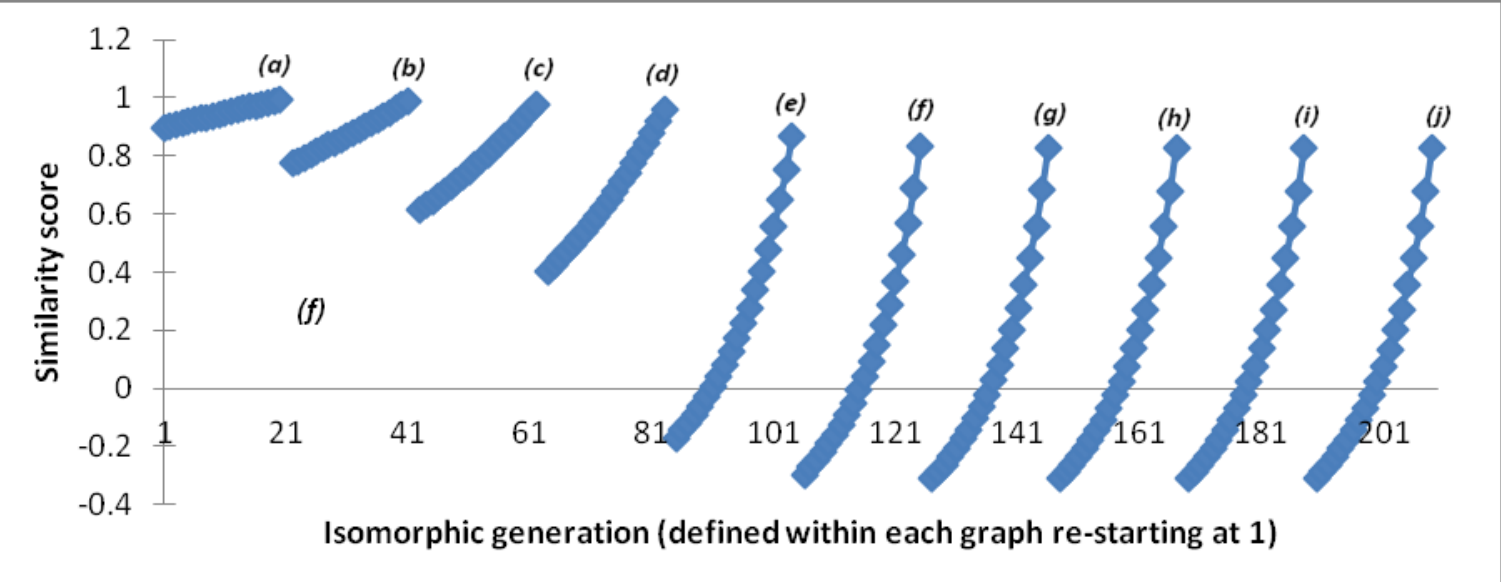

Figure E3: Regression models of the relationship between the generations rank of isomorphs and the translated percentages of optinalysis. (a)-(j) are the regression models at skewization value $t=10,25,50,10^{2}, \ldots \ldots 10^{8}$ respectively. (a)-(d) are linear $\rightarrow$ exponential $\rightarrow$ simple polynomial, $(e)-(j)$ are complex polynomial models. 